of

2. 3 .

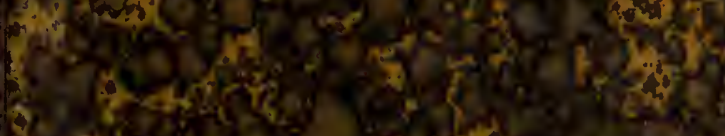

$8 x^{2}, x^{2}=$

की



(2)

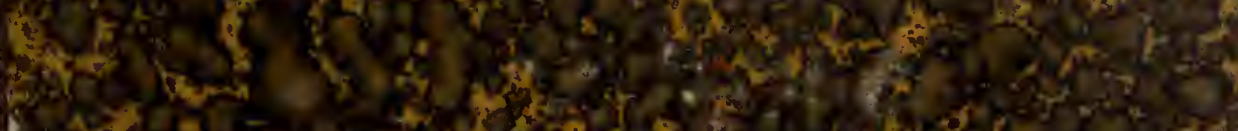

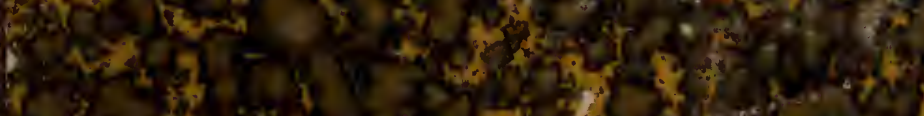

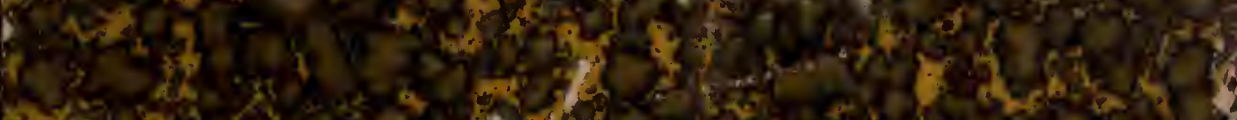

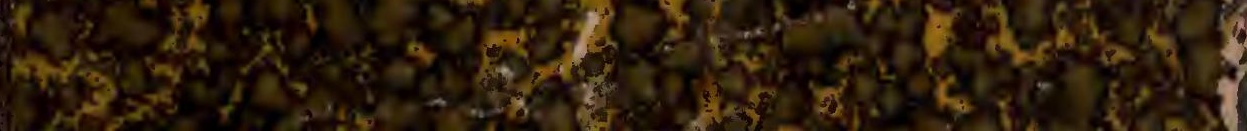
(1)

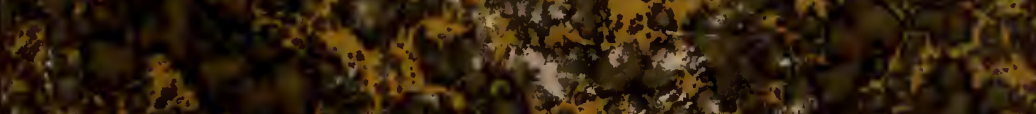

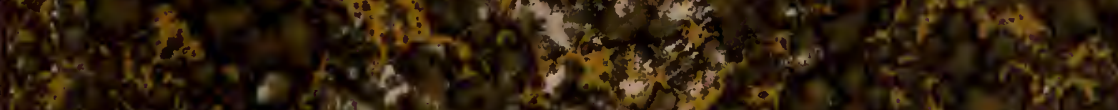

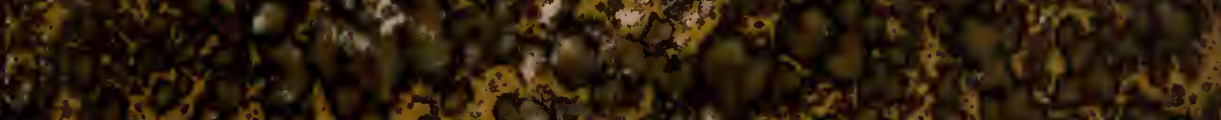

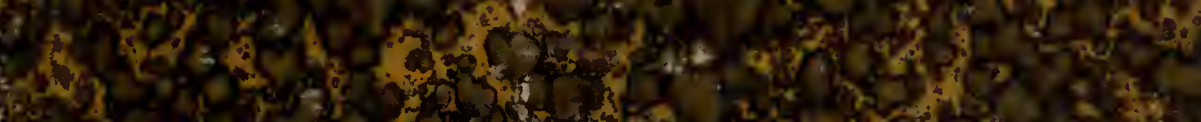

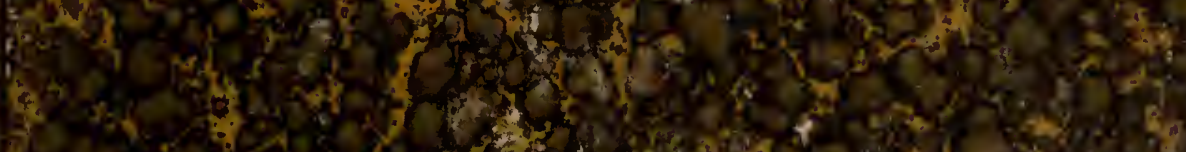

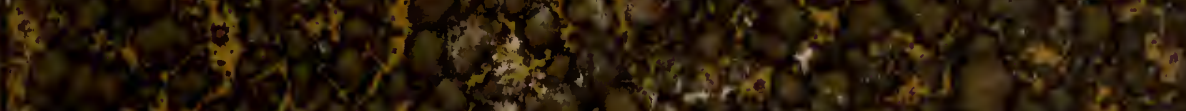

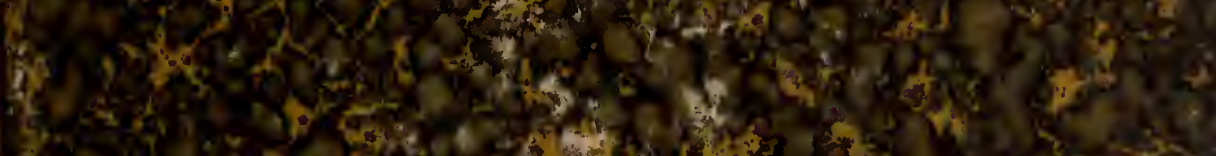

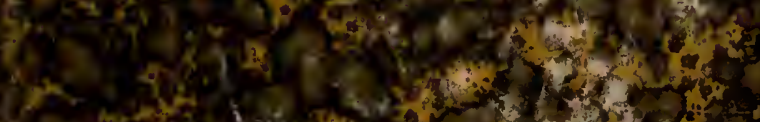

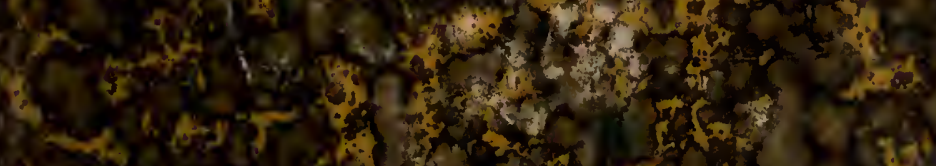

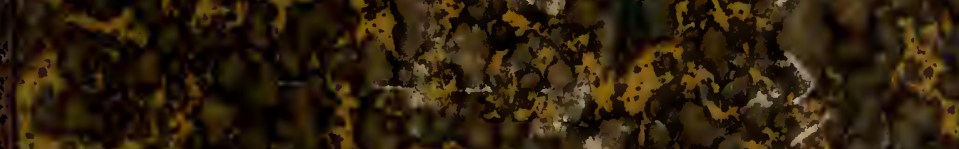

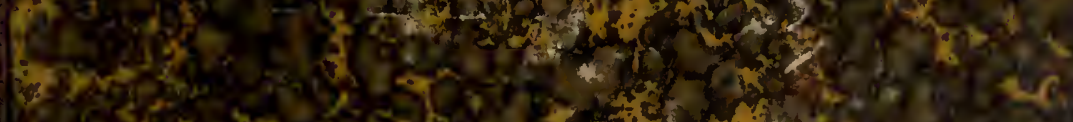

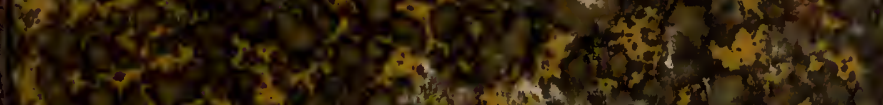

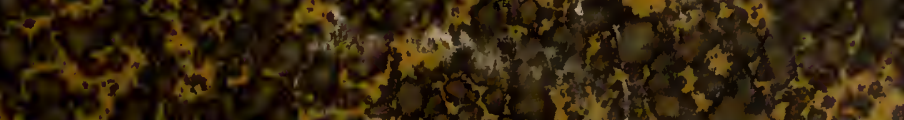

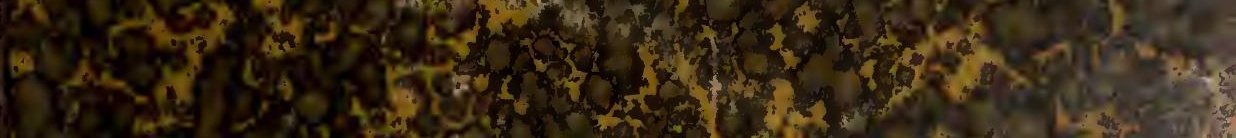

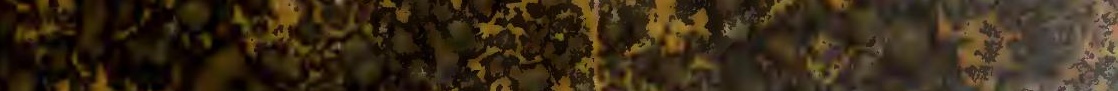

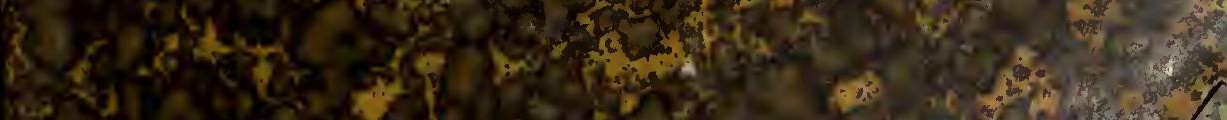

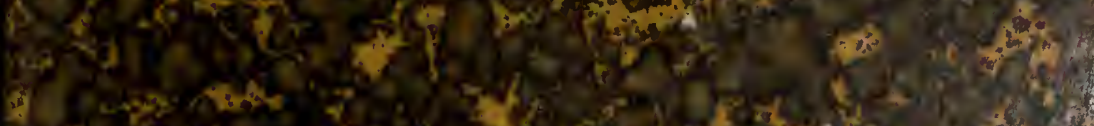






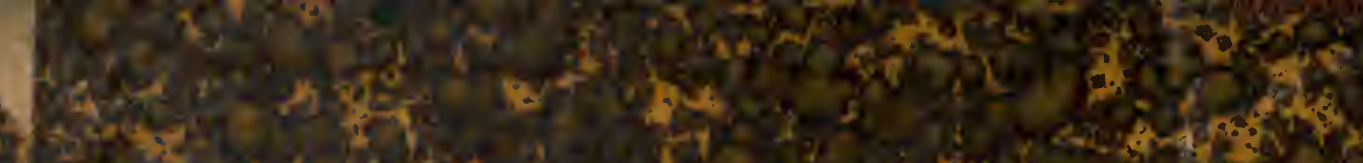

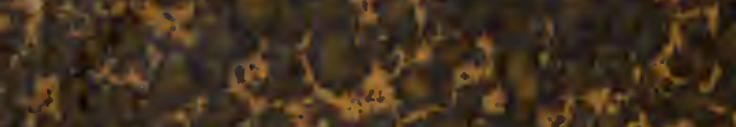
4. 4 a

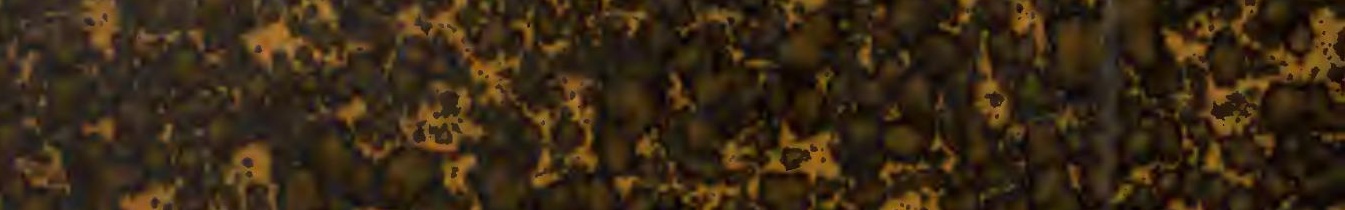

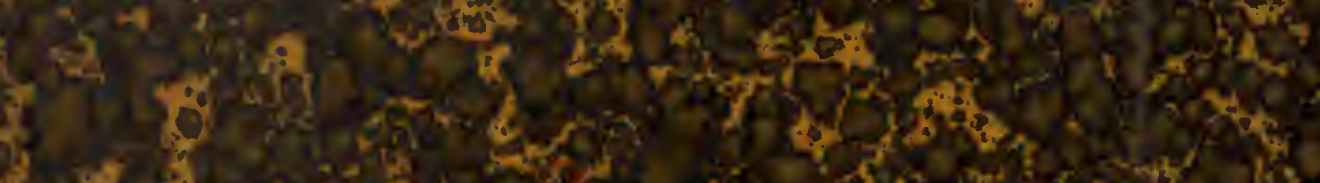

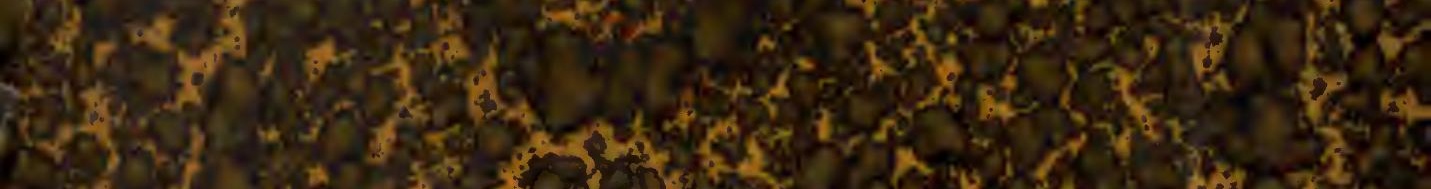
$33 \times 2$. expert?

is

35 3

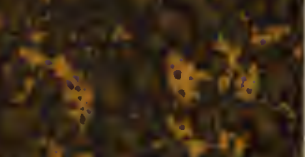

.

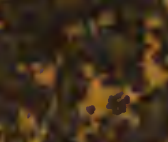

$3 \times$ ses 9.

secte

200
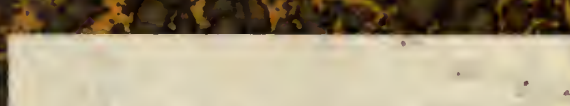

THE UNIVERSITY

OF ILLINOIS

LIBRARY

590.5 F.I

uppainat

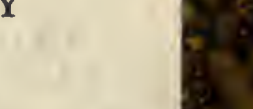

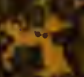

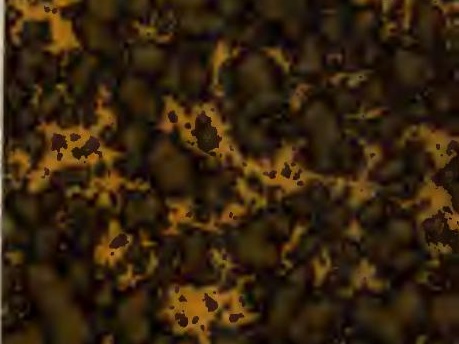

of 34 by

(3)

$3 \times 2$
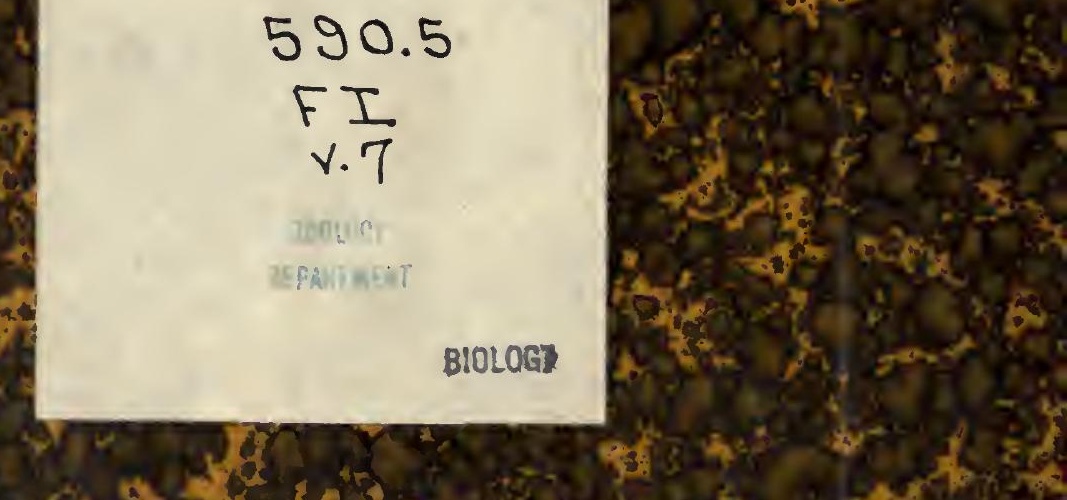

W1



(9) कa $4 x^{2}$

西<smiles>[CaH]</smiles>

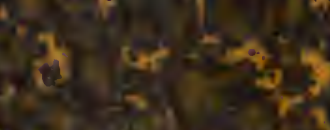

$4 x^{2}$

$261: 9 x^{2}$

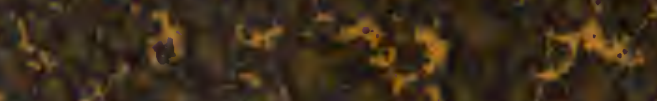

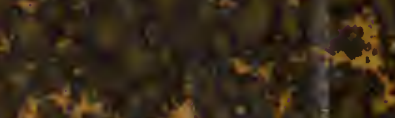

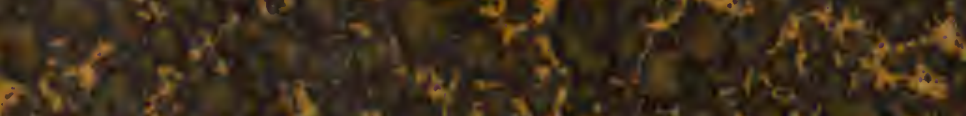







\title{
PUBLICATIONS
}

OF THE

$$
\begin{gathered}
\text { LBBRARY } \\
\text { OF THE } \\
\text { UNIVERITY OO } \\
\text { 31 MAR'YS }
\end{gathered}
$$

\section{FIELD MUSEUM OF NATURAL HISTORY}

\author{
ZOÖLOGICAL SERIES \\ Volume VII
}

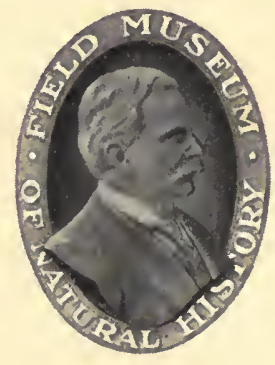

Chicago, U. S. A.

1905-I9I 2 ! 

Field Museum of Natural History.

Publication i6i.

Zoölogical Series.

Vol. VII, No. I3.

\section{CONTENTS AND INDEX}

TO

\section{VOLUME VII, NUMBERS I TO I 2.}

Charles B. Cory,

Curator, Department of Zoölogy.

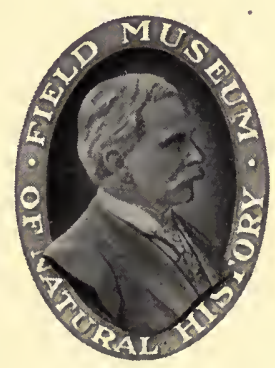

Chicago, U. S. A.

I 9 I 2. 
590.5

F $\frac{T}{7}$

, 


\section{CONTENTS}

Annotated List of a Collection of Reptiles from Southern California and northern Lower California. By Seth Eugene Meek, $\mathrm{Ph} . \mathrm{D} . . . . . .$. .

A Catalogue of the Fishes of Bermuda, with Notes on a Collection made in I 905 for the Field Museum. By Dr. Tarleton H. Bean...........

Description of three New Species of Fishes from Middle America. By

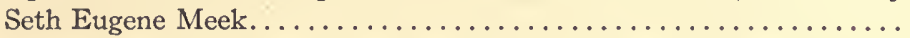

Synopsis of the Fishes of the Great Lakes of Nicaragua. By Seth Eugene

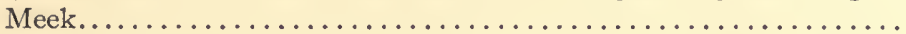

Notes on Fresh-water Fishes from Mexico and Central America. By Seth

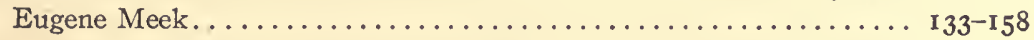

The Zoollogy of Lakes Amatitlan and Atitlan, Guatemala, with Special Reference to Ichthyology. By Seth Eugene Meek........... I59-206

New Species of Fishes from Tropical America. By Seth Eugene Meek... 207-2 I 2

Description of two new Pulmonate Mollusks with a List of other Species from the Solomon Islands, collected by Doctor G. A. Dorsey. By William Healey Dall, D. Sc..................... 21 23-222

A Synoptic List of the Fishes known to occur within fifty miles of Chicago.

By S. E. Meek and S. F. Hildebrand................ 223-338

Notes on Some Cleridæ of Middle and North America, with Descriptions of New Species. By A. B. Wolcott. . . . . . . . . . . . . . 339-402

Batrachians and Reptiles from British East Africa. By Seth Eugene Meek 403-4I4

Notes on Batrachians and Reptiles from the Islands north of Venezuela.

By Seth Eugene Meek...................... 4r5-4i 8 



\section{LIST OF ILLUSTRATIONS}

\section{PLATES}

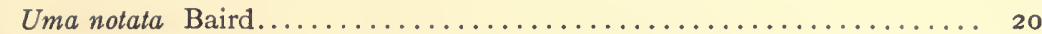

ii Crotalus helleri Meek............................. 20

iii Crotalus willardi Meek............................ 20

iv Placostylus (Leucocharis) dorseyi Dall and Chloritis (Sulcobasis) camaratus

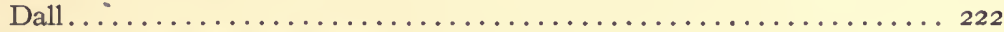

v Fig. I. Cymatodera peninsularis Schaeffer.

"2. Priocera lecontei n. sp.

"3. Thanasimus monticola n. sp.

“4. Trichodes oresterus $\mathrm{n} . \mathrm{sp}$.

" 5. Trichodes nexus $\mathrm{n}$. $\mathrm{sp}$.

“ 6. Isolemidia cariniceps n. $\mathrm{sp}$.

“7. Hydnocera hamatica Gorham.

“ 8. Hydrocerca gorhami n. sp.

“ 9. Cregya granulosa Wolcott...................... 402

vi Figs. I-20. Showing views of last abdominal segments of various species of Cymatodera.

“ 2I-30. Elytral markings of North American Trichodes.

“ 3I. Pronotum of Orthopleura damicornis Fabr.

“ 32. Same of O. texana Bland... 402

\section{TEXT FIGURES}

I. Hippocampus brunneus Bean........................ 39

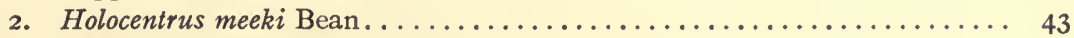

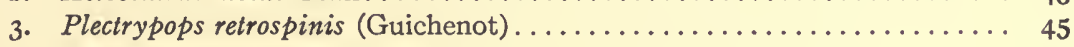

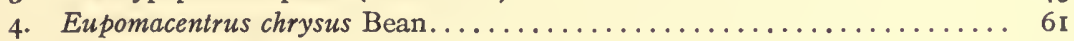

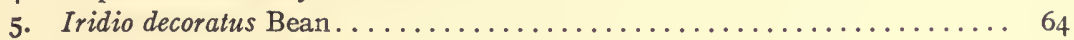

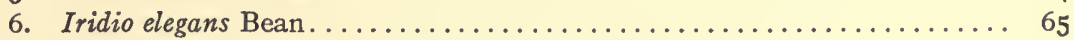

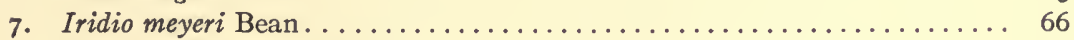



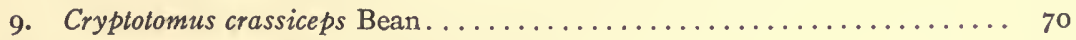

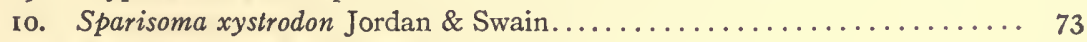

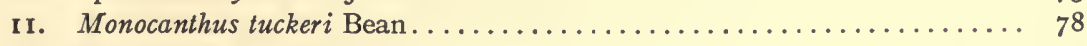

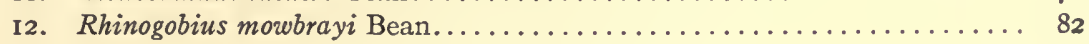


PAGE.

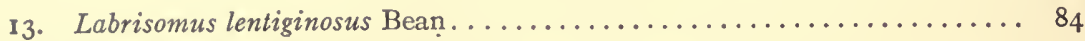

14. Antennarius verrucosus Bean. ...................... 88

15. Large-mouthed Black Bass, Micropterus salmoides (Lac.), showing parts referred to in description of fishes. .................... ror

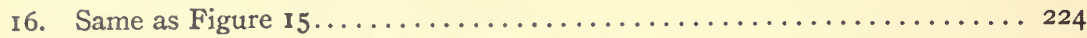

17. Lake Sturgeon. Acipenser rubicundus Le Sueur................ 236

18. Dog Fish; Bowfin; "John A. Grindle." Amiatus calva Linnæus. . . . . . 239

19. Channel Cat; Fiddler. Ictalurus punctatus (Raf.) . . . . . . . . . . . 240

20. Black Bullhead. Ameiurus melas (Raf.)...................... 243

21. Slender Stone Cat. Schilbeodes exilis (Nelson)................ 246

22. Brindled Stone Cat. Schilbeodes miurus (Jordan).............. 247

23. Blunt-nosed River Carp. Carpiodes difformis Cope.............. 250

24. Chub Sucker. Erimyzon sucetta (Lac.).................... 252

25. Lips of the Common Redhorse. Moxostoma aureolum (Le Sueur)....... 253

26. Lips of the White-nosed Sucker. Moxostoma anisurum (Raf.)........ 254

26. Lips of the Short-headed Redhorse. Moxostoma breviceps (Cope)...... 255

27. Common Sucker. Catostomus commersonii (Lac.)............... 256

28. Blunt-nosed Minnow. Pimephales notatus (Raf.)............... 263

29. Small-mouthed Minnow. Opsoperodus emilice Hay ................ 264

30. Golden Shiner; Roach. Notemigonus crysoleucas (Mitch.)........... 265

31. Bullhead Minnow; Flathead. Cliola vigilax (Baird \& Girard)........ 266

32. Least Minnow. Notropis anogenus Forbes.................. 268

33. Black-striped Minnow. Notropis heterodon (Cope)............... 269

34. Blunt-nosed Minnow. Notropis cayuga Meek.................. 269

35. Straw-colored Minnow. Notropis blennius (Girard)............. 270

36. Silvery Minnow. Notropis jejunus (Forbes)................ 27 I

37. Spot-tailed Minnow. Notropis hudsonius (DeW. C.)............. $27 \mathbf{I}$

38. Common Shiner. Notropis cornutus (Mitch.)............... 273

39. Redfin. Notropis umbratilis (Girard).................... 274

40. Silver-mouthed Minnow. Ericymba buccata Cope............... 275

41. Sucker-mouthed Minnow. Phenacobius mirabilis (Girard).......... 276

42. Spotted Shiner. Hybopsis dissimilis (Kirtland).............. 277

43. Bib-eyed Chub; Silvery Chub. Hybopsis amblops (Raf.)........... 278

44. River Chub; Horny-head. Hybopsis kentuckiensis (Raf.).......... 279

45. Horned Dace; Creek Chub. Semotilus atromaculatus (Mitch.)....... 280

46. Long-nosed Dace. Rhinichthys cataracte (C. \& V.)............... 28 I

47. Black-nosed Dace. Rhinichthys atronasus (Mitch.)............. 282

48. German Carp; Leather Carp. Cyprinus carpio Linnæus............. 283

49. Toothed Herring; Mooneye. Hiodon tergisus Le Sueur............ . 287

50. Gizzard Shad; Hickory Shad. Dorosoma cepedianum (Le Sueur) ...... 288

5I. Common Whitefish. Coregonus albus Le Sueur................. 290

52. Ciscoe and Blueback Herring of Lake Michigan. Argyrosomus huronius Jordan \& Evermann. . . . . . . . . . . . . . . . . . . . . 292

53. Blackfin. Argyrosomus nigripinnis Gill................... 293

54. Steelhead; Salmon Trout. Salmo gairdneri Richardson............ 294

55. Mud Minnow. Umbra limi (Kirtland)................... 298

56. Top Minnow. Fundulus notatus (Raf.)................... 300

57. Brook Stickleback. Eucalia inconstans (Kirtland)............... 302

58. Trout Perch. Percopsis guttatus Agassiz.................... 304 
59. Brook Silverside. Labidesthes sicculus (Cope).................. 305

6o. Calico Bass. Pomoxis sparoides (Lac.)........................ 308

61. Warmouth. Chøenobryttus gulosus (C. \& V.)................ 3 I0

62. McKay's Sunfish. Lepomis euryorus McKay................. 312

63. Bluegill; Blue Sunfish. Lepomis incisor (C. \& V.)................ 3 I4

64. Large-mouthed Black Bass. Micropterus salmoides (Lac.)........... 3 I6

65. Wall-eyed Pike; Jack Salmon. Stizostedion vitreum (Mitch.)........ 3 I9

66. Log Perch. Percina caprodes (Raf.)........................... 32 I

67. Black-sided Darter. Hadropterus aspro (Cope \& Jordan)........... 322

68. Shumard's Darter. Cottogaster shumardi (Girard) .............. 323

69. Green-sided Darter. Diplesion blennioides Raf................ 324

70. Blunt-headed Darter. Boleosoma camurum Forbes.............. 325

71. Sand Darter. Ammocrypta pellucida (Baird)................ 326

72. Banded Darter. Etheostoma zonale (Cope) ................. 327

73. Rainbow Darter; Soldier-fish. Etheostoma cœruleum Storer.......... 329

74. Spindle-shaped Darter. Boleichthys fusiformis (Girard)............ 33 I

75. Least Darter. Microperca punctulata Putnam................. 332

76. Yellow Bass; Striped Bass. Morone interrupta Gill............... 334

77. Common Sculpin; Miller's Thumb. Cottus ictalops (Raf.) . . . . . . . 336

\section{TEXT VIEWS}

Fishing for Sardinas........................... I 5

Volcan de Agua from Lake Amatitlan. . . . . . . . . . . . . . . . I60

Hotel Laguna, Lake Amatitlan. ........................ I6I

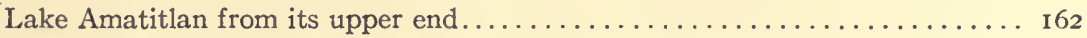

Shore near outlet of Lake Amatitlan. . . . . . . . . . . . . . . . . . I63

Delta at mouth of Lobos River, Lake Amatitlan . . . . . . . . . . . I63, I64

Contour map of Lake Amatitlan.......................... I 65

Hot Spring, Laguna, Lake Amatitlan..................... I66

Willows on upper portion of Lake Amatitlan................... I69

Upper end of Lake Amatitlan. . . . . . . . . . . . . . . . . . . I69

Wild lettuce, Lake Amatitlan............................ I 7 I

Fishermen, Lake Amatitlan. ....................... I 77

Lake Atitlan, showing depth where soundings were made........... I79

Lake Atitlan near Panajachel........................... I 8 I

Fish Trap, Lake Amatitlan............................ I 85

Fish Runways, Santa Catarina, Lake Atitlan................... I 87

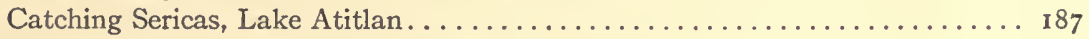

Fishing for Mojarras, Lake Amatitlan..................... I90 



\section{ERRATA}

Pages 142 and 15I, and line from bottom, for Cichlasoma montaguensis, read Cichlasoma motaguensis.

Page I92, Ioth line from bottom, for Basaliscus vittatus, read Basiliscus vittatus. Page 199, 4th line from top, for Glossophonia lineata, read Glossiphonia lineata. Page 323, under Fig. 68, for Cottagaster shumardi, read Cottogaster shumardi.

Page 326, under Fig. 7I, for Ammocyipta pellucida, read Ammocrypta pellucida.

Page 35I, 5 th line from bottom, for a cotype $\left(\Omega^{\top}\right)$, read a cotype ( + ).

Page 362, 6th line from bottom, for Systenoderus dastoides, read Systenoderus dasytoides.

Page 362,4 th line from bottom, for Pacilichroa Haagi, read Pacilochroa Haagi.

Page 379, 6th line from top, for Rev. Henry Steven Gorham, read Rev. Henry Stephen Gorham.

Page 4I6, I4th line from top, for Oxbelis accuminatus, read Oxybelis acuminatus. 



\section{INDEX.}

\begin{tabular}{|c|c|}
\hline & \\
\hline Abildgaardi, Sparisoma......... 7 I & albus, Coregonus............ \\
\hline Ablepharus wahlbergii....... 412 & Alcedinidæ.............. I96 \\
\hline Abudefduf saxatilis........... 60 & alcyon, Ceryle............ 196 \\
\hline Acacia............... 392 & alfari, Cichlasoma ......... I 48 \\
\hline Acanthopteri........... 301 & Algæ.............. I 80 \\
\hline acanthura, Ctenosaura......... I92 & Alleterata, Gymnosarda....... 46 \\
\hline Acipenser. . . . . . . . 30, 36, 235 & alligator, Anolis........... 4 I 6 \\
\hline Acipenser rubicundus. . . . . . 236 & alliteratus, Orcynus......... 46 \\
\hline Acipenseridæ........... 30,235 & Alona................. 204 \\
\hline Acititis macularius. . . . . . . I 95 & alosoides, Hiodon........... 286 \\
\hline acuminatus, Eques......... 60 & Alphestes chloropterus....... $\quad 55$ \\
\hline Myrichthys........... 3 I & altum, Sicydium.......... I 49 \\
\hline Oxybelis............ 416 & Alutarius scriptus.......... 77 \\
\hline acus, Tylosurus........... 35 & 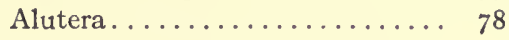 \\
\hline acutum, Cichlasoma......... I5 I & schœpffi.............. \\
\hline acutus, Pseudoscarus........ 70 & scripta........... 77 \\
\hline Scarus............ 70 & Ambloplites............ 309 \\
\hline adamsi, Bullaria.......... 217 & rupestris ........ I79, 309 \\
\hline Adelphoclerus............. 356 & amblops, Hybopsis. . . . . . . 278 \\
\hline nitidus............ 357 & Ameiurus............... 24 I \\
\hline adonis, Papuina........... 215 & lacustris............242 \\
\hline adustus, Eupomacentrus....... 6I & melas................ 243 \\
\hline æmula, Cymatodera......... 348 & natalis............. 242 \\
\hline æneicollis, Clerus......... 360 & nebulosus...........242 \\
\hline æneus, Astyanax. . . . 108, I 34, I 45, I 50 & Ameiva surinamensis......... 4I7 \\
\hline Tetragonopterus......... I35 & undulata .......... \\
\hline æstivalis, Hybopsis.......... I 55 & americana, Fulica.......... 195 \\
\hline æthiops, Cymatodera......... $35^{\circ}$ & Amiatidæ............... 238 \\
\hline Aëtobatus narinari......... 30 & Amiatus................ 238 \\
\hline affinis, Gambusia.......... I 55 & calva ............... \\
\hline Agama atricollis........... 408 & Ammocrypta............ 326 \\
\hline caudospinosa........ 407 & pellucida........... \\
\hline colonorum.......... 407 & Amnicola guatemalensis . . . . . 206 \\
\hline agassizii, Gopherus........... & petensis.......... \\
\hline Scorpæna............. 80 & Amnicolidæ........... \\
\hline Agonostomus monticola..... I39, 147 & Anabæna.............. 172 \\
\hline albomaculata, Priocera........ 384 & Anacanthini........... 337 \\
\hline Albula vulpes............ 33 & Anacystis............. I 72 \\
\hline Albulidæ................ 33 & 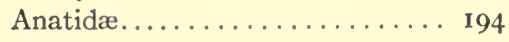 \\
\hline album, Hæmulon........... $5^{8}$ & anchovia, Sardinella........ 34 \\
\hline albuquerquensis, Diaptomus.... 205 & Ancylus excentricus......... 206 \\
\hline & \\
\hline
\end{tabular}




\section{Field Museum of Natural History-Zoölogy, Vol. ViI.}

PAGE.

Angelichthys ciliaris . . . . . 74, 75

formosus............ 74

Anguidæ............... 12

anguiformis, Sphagebranchus.... 3 I

Anguilla................. 284

chrysypa............ 30

rostrata............ 285

Anguillidæ................30, 284

angulifera, Cymatodera . . . . . . 354

angustifolia, Typha.......... I 70

anisurum, Moxostoma........ 254

annectens, Gambusia . . . . . . . . I 145

Anniella pulchra............ I3

Anniellidæ.............. I3

annularis, Pomoxis. . . . . . . . 308

anogenus, Notropis... . . . . . . . 268

Anolis alligator............ 416

lineatus............. 4 I6

anomalum, Campostoma.... . . 260

Antennariidæ............. 87

Antennarius............. 87

nuttingii........... 87

principis............ 89

radiosus............. 89

scaber.............. 87

stellifer.............. 89

verrucosus............ 88

antennata, Cymatodera....... 346

antiquorum, Hippocampus..... 40

Pristis............. I04

Aphredoderidæ............ 305

Aphredoderus.............. 306

apivorus, Trichodes....370, 371, 375

sayanus............ 306

apivorus var. interruptus, Trichodes....................... 376

Aplodinotus. . . . . . . . . . . 334

grunniens............ 335

Apodes................... 284

apodus, Lutianus..... . . . . . . . 55

Apogon binotatus.......... 5 I

imberbis............ 5I

maculatus........... 5 I

pigmentarius.......... 50

sellicauda........... 5 I

aramaca, Hemirhombus. ....... 87

Archocentrus............. I26

Ardeidæ................... 194

aredola, Tylosurus........... 36
PAGE.

arenatus, Priacanthus ........ 55

arge, Chirostoma............ I56

argenteus, Diplodus.......... 59

argentiventris, Neomænis. . . . . . . I40

Argyrosomus............. 29I

hoyi.............. 292

huronius............29I

nigripinnis............ 293

prognathus........... 292

arietans, Bitus............ 405

arizonæ, Cymatodera. . . . . . . . 349

Arthroleptis minutus.... . . . . . 403

Asaphia deflorata........... 2 I9

ascensionis, Holocentrus....... 42

aspera, Hydnocera. . . . . . . . . . . 379

asperella, Cancellaria. . . . . . . 2 I 7

aspro, Hadropterus. . . . . . . . . 32 I

Astyanax................ 107

æneus........ I08, 134, 145, I50

mexicanus. . . . . . . I53, I55

micropthalmus.......... I84

nasutus............. I08

œrstedii.............. I 45

regani............. 207

Asymmetron lucayanum. . . . . . 29

atæniatus, Chætodon......... 73

ater, Faunus.............. 2 I 7

Sauromalus........... 9

Atherina harringtonensis....... 40

Atherinidæ. . . . 40, I I 4, I 39, I 56, 304 atherinoides, Notropis..... . . . 273

Athlennes hians............ 36

atlanticus, Tarpon.......... 33

Atractosteus tropicus.......... 104

atricollis, Agama........... 408

atripinnis, Goodea... . . . . . . I 53, I56

atromaculatus, Semotilus....... 280

atronasus, Rhinichthys....... 28 I

atrox, Crotalus............. I8

Aulicus monticola . . . . . . . 364, 365

nero.............. 364, 365

Aulostomidæ............ 37

Aulostomus maculatus. . . . . . . . 37

aurantiacus, Ceratacanthus..... 76 aurantia, Nerita........... 218 auratus, Carassius. . . . . . 30, I44, 284 aureolum, Moxostoma........ 253 aurofrenatum, Sparisoma....... 72 aurolineatum, Bathystoma..... $5^{8}$ 
aurorubens, Rhomboplites...... 26

Auxis rochei............. 46

thazard............ 46

aya, Lutianus............ 57

badeni, Clerus. . . . . . . . . . . 359

bahianus, Teuthis........... 75

bahiensis, Exocœtus........ 37

baileyi, Crotaphytus......... 8, 9

bajonado, Calamus.......... 59

Balistes carolinensis......... 76 vetula............ 76

Balistidæ.............. 76

balsanus, Melaniris........... I I 4

balteatum, Cichlasoma . . . I29, I30, I49

balteatus, Heros.............. I29

barbata, Brotula........... 86

Rhamdia............. I06

Barbatia................ 219

barracuda, Sphyræna........ 4 I

bartholomæi, Caranx......... 46

Bascanium flagellum.......... I5

Basiliscus vittatus. . . . . . . . I92

basilaris, Heros............. 122

bathyraphe, Clanculus........ 218

Bathystoma aurolineatum..... 58 rimator........................... $5^{8}$

striatum............ 58

- Batrachia.............403,4 15

belizanus, Belonesox.......... I5I

belliana, Kinixys........... 4 4

Belonesox belizanus.......... I 5 I

bermudæ, Fundulus... . . . . . 26, 35

bermudarum, Calionymus...... 8 8 I

bermudensis, Fierasfer........ 85

Lefroyia............. 85

bibalteatus, Trichodes......370, 37 I

bicolor, Clerus............. 359

bifasciata, Phrynomantis.... . . 404

bifasciatus, Chlorichthys ....... 68

bilineicolle, Cregya.......... 393

Pelonium............ 392

bimaculata, Orthopleura... . . . . 395

bimaculatus, Clerus ......... 359

Pseudociphorus.......... I5 I

Trichodes............ 374

binotatus, Apogon.......... 5 I

bioculatus, Clerus. . . . . . . 359, 360

biseriatus, Sceloporus. . . . . . . . . I I I
PAGE.

bisignatus, Trichodes........ 374

bistrispinus, Rhypticus....... 55

Bithynis jamaicensis........ 202

Bitis arietans............ 405

bivittatus, Iridio.......... 63

blainvillii, Phrynosoma........ I 12

Blenniidæ.............. 83

blennioides, Diplesion........ 323

Blennius cristatus.......... 85

blennius, Notropis.......... 270

bocagei, Hylambates......... 404

boddærti, Drymobius......... 416

Bodianus fulvus........... 52

fulvus punctatus....... 52

fulvus ruber......... 52

Boleichthys.............. 330

fusiformis.......... 331

Boleosoma................ 324

camurum............ 324

nigrum............. 324

bombycinus, Clerus......... 360

bonaci, Mycteroperca......... 53

bonariense, Hæmulon. . . . . . . . $5^{8}$

borealis, Sphyræna.......... 42

Bosmina obtusirostris......... 204

Bosminidæ... . . . . . . . . . . . 204

bourgainvillei, Chloritis........ 220

Chloritis (Sulcobasis)..... 2 I 6

bowersi, Mycteroperca........ 54

boylii, Lampropeltis. . . . . . . . . I 5

brachycephalus, Rhamdia...... . I44

brachyops, Paludicola......... 4I 5

brachypterus, Colymbus domini-

cus................. I94

brachyurum, Diaphanosoma... . 203

Brama raii.............. 50

Bramidæ................ 50

Bramocharax.............. I Io

bransfordi............ I Io

elongatus............. I Io

Branchiostoma caribxum....... 29

Branchiostomidæ............ 29

bransfordi, Bramocharax........ I Io

brasiliensis, Hemirhamphus..... 36

Mugil............. 4I

braytoni, Notropis............ I 55

breviceps, Moxostoma......... 254

brevicollis, Mabuia........... 4 Io

bricei, Chætodon............ 74 
brookii, Hemidactylus........ 406

Brosmophycis verrilli......... 86

Brotula barbata........... 86

Brotulidæ............... 85

brunneus, Hippocampus...... 39

Brycon................. 109

dentex....... I09, I I 2, I 35, I 52

bubalus, Ictiobus........... 249

buccanella, Lutianus......... 57

buccata, Ericymba.......... 275

Bufo marinus............. I9I

regularis............ 404

Bufonidæ............... I9 I

Bullaria adamsi............ 217

bullatus, Tectarius......... 218

butleri, Poecilia............ I38

Butorides virescens.......... I94

caballus, Caranx....... 48

cabreræ, Rhamdia.......93, 134, 183

Calamus calamus............ 59

bajonado............ 59

calamus, Calamus ......... 59

calientis, Notropis......... 153: 155

californica, Cymatodera.....349, 350

Monophylla.....340, 341, 342

californicus, Colymbus nigricollis. 194

Elasmocerus......... $34 \mathbf{I}$

californiensis, Eucinostomus... . . I 43

Calionymus bermudarum....... 8 $8 \mathrm{I}$

callichloros, Leptopoma........ 216

Callionymidæ............. $8 \mathbf{I}$

Callisaurus ventralis......... 7

calva, Amiatus............ 238

camaratus, Chloritis (Sulcobasis)

$$
\text { .............216,220 }
$$

campbelli, Strombus......... 217

Campostoma............. 260

anomalum............ 260

camurum, Boleosoma........ 325

canadense, Stizostedion....... 318

canadus, Rachycentron....... 49

Cancellaria asperella......... 217

candida, Placostylus pancheri.... 2 I9

canis, Mustelus............ 29

Canthidermis maculatus....... 76

Canthigaster rostratus.......26, 80

Canthigasteridæ........... 80

capense, Lycophidum........ 405
PAGE.

capistratus, Chætodon........ 74

caprodes, Percina........... 320

Carangidæ.............. 46

Caranx bartholomæi.......... 46

caballus............ 48

chrysos............. 47

guara.............. 47

latus.............. 47

ruber.............. 48

carapo, Gymnotus......... I 35, 184

Carassius............................ 283

auratus.......... $30,144,284$

Carassius carassius.......... 283

carassius, Carassius.......... 283

carbonarium, Hæmulon....... 57

Carcharhinus............. I03

lamia............. 29

nicaraguensis.......... I03

platyodon............ 29

Cardinalia virgata.......... 218

caribæum, Branchiostoma...... 29

cariniceps, Isolemidia........ 376

carolina, Porzana............ 195

carolinensis, Balistes......... 76

carpintus, Neetroplus......... 156

carpio, Cyprinus........... 282

Carpiodes................ 250

difformis............ 250

elongatus............ 154

microstomus......... 154

velifer.............25 I

Cassina senegalensis......... 404

castanea, Priocera............... 356

cataractæ, Rhinichthys........ 28 I

catenata, Echidna.......... 32

catenifer, Pityophis.......... I5

Catorama.......................... 364

Catostomidæ........... I54, 248

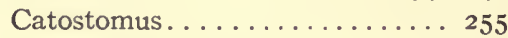

catostomus........... 255

commersonii..........256

nigricans........... 257

catostomus, Catostomus....... 255

caudata, Poecilia........... 209

caudospinosa, Agama........ 407

Causus resimus............ 405

cayorum, Ogilbia........... 85

cayuga, Notropis............ 269

centrale, Cichlasoma........94, 140 
PAGE.

Centrarchidæ...........156, 306

centrarchus, Cichlasoma........ 126

Heros.............. 126

Centriscus scolopax........... 38

Centrolophidæ............. 50

Centrolophus.............. 50

Centropagidæ.............. 205

Centropomidæ............. I40

Centropomus nigrescens.....140, 174

cepedianum, Dorosoma........ 287

Cephalacanthidæ........... 8r

Cephalacanthus volitans...... 8I

cerastes, Crotalus........... 18

Ceratacanthus aurantiacus..... 76

Ceratophyllum.............. I86

demersum........... I 7 I

Ceriodaphnia rigaudi........ 204

Cerithium morus............ 217

Ceryle alcyon.............. 196 americana septentrionalis... 196

Chænobryttus............... 3 I0 gulosus............... 310

Chætodon atæniatus.......... 73 bricei............... 74

capistratus............. 74

striatus.............. 74

Chætodontidæ............. 73

Chalcinopsis dentex........... I09

Chamæleon dilepis........... 4 I 4 ellioti............... $4^{\mathrm{I} 4}$

hoeneli............... 4 $\mathrm{I}^{\mathrm{I}} 3$

jacksoni.............. $44^{13}$

Chara.............171, 180, I86

Characinidæ........ 107, 134, I45, I50, $\ldots \ldots \ldots \ldots \ldots 152,155,184$

Characodon variatus.......... I55

Charadriidæ............... 196

Chariessa................... 383

dichroa.............. 383

elegans................ 383

pilosa..............383, 394

pilosa var. onusta . . . . . 394

ramicornis............. 383

texana................ 382

vestita............... 383

chavesi, Dorosoma........... I1 2

Cheilodipteridæ............. 50

Chilomeniscus fasciatus......... I4

Chilomycterus reticulatus...... 80

Chilopsis............... 7

Chioractis occipitalis.......... I5

Chirostoma arge............ 156

guatemalensis.......... I I 4

jordani.............. II6

chisoyensis, Pœcilia.......... $\mathbf{3} 8$

Chlorichthys bifasciatus....... 68

nitidus............. 68

Chloritis bourgainvillei........ 220

quercinus...........216, 220

(Sulcobasis) bourgainvillei. . 216

(Sulcobasis) camaratus. . 216, 220

Chlorophis neglectus......... 406

chloropterus, Alphestes....... 55

chœrostomus, Stolephorus...... 34

Chondrostei.............. 235

Chonophorus transandeanus..... 150

Chrosomus................ 260

erythrogaster..........26r

chrysochloris, Pomolobus....... 288

Chrysomelidæ............. 396

chrysops, Roccus............ 333

chrysopterus, Orthopristis...... 58

chrysos, Caranx............ 47

chrysurus, Glyphidodon........ 63

Microspathodon......... 62

Ocyurus.............. 57

chrysus, Eupomacentrus....... 6r

chrysypa, Anguilla.......... 30

Cichlasoma............117, 157

acutum............. 15I

alfari............... 148

balteatum........ 129, I30, I49

centrale.............94, 140

centrarchus............ 126

citrinellum...98, 99, 121, 152, 156

dorsatum............. 1 $\dot{2}_{3}$

dovii...............120, I43

ellioti.............. 142 $^{2}$

erythræum.......... 99, 124

fredrichsthalii.......... 143

frontale............. 2 10

granadense........... I21

guttulatum..........141, 89

hedricki............. I5I

labiatum............99, 124

lobochilus..........124, 125

longimanus.......127, 128, 153

macracanthum.......... $\mathbf{r}_{40}$ 


\section{Field Museum of Natural History-Zoölogy, Vol. VII.}

\section{PAGE.}

Cichlasoma-Continued

maculicauda......... I4I, I5I

managuense. . . II9, I 43, I 52, I 75

manana............. I4I

milleri.............. I 42

mojarra........... I40, I4I

montaguense...... 142, I 51 , I 74

nicaraguense........ I29, I30

nigritum........... I 28

nigrofasciatum..... I4I, I80, I 89

punctatum........... 210

rostratum.......126, I28, I52

spilurum.......... I5 I

tenue.............. 94

trimaculatum.93, 94, 95, 140, 174

underwoodi........... 2 I I

zonatum........... I4 I

Cichlidæ....... II 7, I40, I48, I5I, I52,

$\ldots \ldots \ldots \ldots \ldots \ldots$ 156, 189

ciliaris, Angelichthys.........74, 75

ciliatus, Monacanthus......... 77

cinctipennis, Macrotelus....... 34 I

Monophylla.........340, 34 I

cinctiventris, Rappia......... 404

cinereum, Xystæma......... 60

Cinosteridæ............. 193

Cinosternum cruentatum...... 193

cirratum, Ginglymostoma...... 29

citrinellum, Cichlasoma... 98, 99, I 2 I, $\ldots \ldots \ldots \ldots \ldots \ldots{ }^{I_{52}}$

Cladocera Sueciæ. . . . . . . . . . 204

Clanculus bathyraphe......... 2 I 8

clarkii, Sceloporus........... I I

Clathrocystis............ I 72

clemensæ, Vivipara......... 22 I

Clemmys marmorata......... 3

Cleronomus................. 363

ornaticollis........... 362

Clerosoma............... 363

Clerus..........356, 357, 359, 396

æneicollis............ 360

badeni............... 359

bicolor.............. 359

bimaculatus.......... 359

bioculatus..........359, 360

bombycinus............. 360

cuprescens............. $35^{8}$

cyanipennis.......358, 362, 363

dasytoides........... $35^{8}$
PAGE.

Clerus-Continued

decussatus.............36r

decussatus var. ornatus.... 36I

erythrogaster........... 358

haagi................. 358

Hopfneri............ 36r

latefasciatus.......... 360

laticinctus............ 362

lecontei............. 359

lunatus................... 359

mutillarius........... 357

nigriventris.......... 359

Nuttalli............. 374

oculatus......................... 389

opifex............... 360

quadrisignatus.......359, 362

rufiventris........... 358

salvini............. 361

sphegeus............. 358

(Thaneroclerus) tantillus... 396

thoracicus................ 358, 362

viduus.................. 358

vulneratus........... 362

Cliola.................. 265

vigilax............. 266

Clupeidæ...............33, 288

Cnemidophorus ............ 8

lemniscatus.......... $4 \mathrm{I} 7$

murinus.................... 417

nigricolor............ $4 \mathrm{I} 7$

stejnegeri........... I4

tigris.............. I3

Cœlastrum microporum....... 196

cœruleum, Etheostoma........ 329

cœruleus, Scarus........... 69

Teuthis............ 75

Coleonyx variegatus......... 3

colonorum, Agama.......... 407

Colubridæ.............. I4

Colymbus dominicus brachypterus 194

nigricollis californicus..... 194

comans, Cymatodera......... 35I

commersonii, Catostomus...... 256

concolor, Ichthyomyzon....... 232

congestum, Moxostoma........ I 54

Copepoda................ 205

Coregonus.................290

albus............... 290

quadrilateralis......... 29I 
cornea, Neritina........... 2 I 8

cornutus, Notropis... . . . . . 272

coronatum, Phrynosoma....... I2

Coronella semiornata . . . . . . . 406

Corynetes marginellus. . . . . . . . 397

pallipes.............. . 397

Coryphæna hippurus. . . . . . . . 49

Coryphænidæ.............. 49

Cottidæ................ 335

Cottogaster.............. 322

shumardi........... 322

Cottus................. 335

ictalops............ . 336

ricei................ 336

crassiceps, Cryptotomus........ 70

Cregya..............383, 384, 385

bilineicolle... . . . . . . 393

fasciata.........386, 389, 390

granulosa............. 386

leucophæa.....384,385,386, 388

leucophæa (vetusta)..... 390

maculicollis.......386, 388, 390

$\operatorname{mixta} \ldots \ldots \ldots \ldots 390,391,392$

oculata......386, 389, 390, 39 I

quadrinotata........... 393

quadrisignata..... . 390, 392, 393

vetusta.........384,388, 390

cristatus, Blennius............ 85

Cristivomer... . . . . . . . . . . . 294

namaycush... . . . . . . . . 294

croicensis, Scarus... . . . . . . . . 69

Crotalidæ................. I6

Crotalus................ 3

atrox.............. I 8

cerastes............. I 8

lepidus............. I9

lucifer............... I 7

helleri.............. 17

mitchellii............ I 8

ruber................. I7

terrificus............ 4I6

tigris............. I6

willardi............ 18

Crotaphytus baileyi........ 8, 9

silus............... 9

wislizenii............ 8,9

cruentatum, Cinosternum...... I93

cruentatus, Petrometopon...... 54

Priacanthus.......... 55
PAGE.

crumenophthalmus, Trachurops.. $4^{8}$

Cryptonthe.............. 7

Cryptotomus crassiceps........ 70

crysoleucas, Notemigonus. . . . . . 264

Ctenosaura acanthura......... I92

cuprescens, Clerus........... $35^{8}$

curema, Mugil............ 4 I

cyanea, Furcaria............ 63

cyanellus, Lepomis........... 3 3I I

cyanipennis, Clerus.... . 358, 362, 363

Pœcilochroa........... 362

cyanocephalus, Iridio. . . . . . . . 68

cyanoguttatum, Cichlasoma . . . . I56

Cycleptus elongatus. . . . . . . I 53, I 54

Cycloganoidea............. 238

Cyclopidæ................. 205

Cyclops oithnoides. . . . . . . . 205

cylindricollis, Cymatodera. . 346, 347

Cymatodera æmula.......... 348

æthiops ............ 350

angulifera........... 354

antennata........... 346

arizonæ............. 349

californica.......... 349, $35^{\circ}$

comans............. 35 I

cylindricollis... . . . . 346, 347

discoidalis.......... 355, 356

duplicata...........353, 354

fascifera............. 348

fuchsii............ $35^{2}$

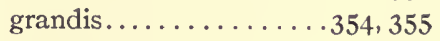

grossa.............. 355

hopei.............. 350

horni............ 349, $35^{\circ}$

isabellæ............. 345

lævicollis............ 345

longicornis............ 343

pallida............... 344

peninsularis.......... 346

santarosæ.......... 35 I

snowi............... 349

soror.............. 342

subsimilis........... 343

torosa............... 347

tuta............... 344

uniformis............ 344

usta . $342,343,344,346,347,348$

wickhami........... 354, 355

xanti.............. 345 
PAGE.

Cynodontichthys tenuis....... I45

Cypræa isabella............ 217

$\operatorname{lynx} \ldots \ldots \ldots \ldots \ldots \ldots 217$

Cyprinidæ..........30, I44, I54, 257

Cyprinodon dearborni......... 208

Cyprinus............... 282

carpio............. 282

riverendi............ 208

Cystignathidæ........... I92

damicornis, Orthopleura. . . 394, 395

Tillus................ 395

Daphnia galeata........... 204

longispina........... 204

pulex................204

Daphnidæ.............. 204

dasytoides. Clerus........... 358

dasytoides, Systenoderes...... 362

dearborni, Cyprinodon......... 208

Decapterus punctatus......... 48

scombrinus........... 48

decoratus, Iridio........... 64

decussatus, Clerus............ 36r

deflorata, Asaphia.......... 219

delicata, Gallinago........... 195

demersum, Ceratophyllum..... I I I

dendriticum, Siphostoma...... 39

dentex, Brycon...... 109, I 1 2, I35, I 52

Chalcinopsis.......... I 09

Dermatolepis inermis........ 55

Diaphanosoma brachyurum..... 203

Diaptomus albuquerquensis..... 205

dichroa, Chariessa........... 383

difformis, Carpiodes......... 250

dilepis, Chamæleon......... 4 44

Dina microstoma........... 200

Diodon hystrix............ 80

novem-maculatus....... 80

Diodontidæ.............. 80

Diospyros............... 392

Diplesion............... 323

blennioides................ 323

Diplodus argenteus......... 59

Dipsosaurus dorsalis......... 3

discoidalis, Cymatodera....355, 356

discoidea, Hydnocera. . . . . . . 379

discors, Querquedula.......... 194

dispar, Fundulus............ 299

dissimilis, Hybopsis......... 277
PAGE.

Dolichopsis.............. 396

dolomieu, Micropterus........ 316

Dormitator maculatus........ I43

dormitor, Philypnus..... I3 I, I53, I74

Dorosoma............. I I 2, 287

cepedianum.......... 287

chavesi.............. II

exile............... 155

Dorosomidæ......... I I 2, I 55, 287

dorsalis, Dipsosaurus......... 3

dorsatum, Cichlasoma........ 123

dorseyi, Placostylus.........216, 2 I9

Placostylus (Leucocharis) . . 2 I9

Douglassianus, Trichodes...... 373

dovii, Cichlasoma......... I20, I43

Heros.............. I 20

Poecilia ........... I I 3, I $_{3} 8$

drummond-hayi, Epinephelus.... 53

Drupa ricinus............ 217

Drymobius boddærtii......... 416

dubius, Thanasimus......... 357

ductor, Naucrates.......... 48

duplicata, Cymatodera......353, 354

duquesnii, Placopharynx... 248

Echeneididæ............. 83

Echeneis naucrates......... 83

Echidna catenata........... 33

Edwardsii, Trogodendron....... 366

edwardsii, Xenoclerus.......... 366

Elapechis guentheri.......... 405

Elasmocerus............. 339

californicus............ 34 I

megatoma.......... 342

terminatus........... 342

elegans, Chariessa............. $3^{83}$

Eudorina............. 197

Iridio................ 65

Eleocharias.............. 170

Eleotris longiceps........... I3

ellioti, Chamæleon.......... 4I4

Cichlasoma........... I42

elongatus, Bramocharax........ I I

Carpiodes............ 154

Cycleptus.......... 153, 154

Elopidæ............... 33, I I I

elucens, Siphostoma......... 38

emiliæ, Opsopœodus......... 263

Engraulididæ............ 34 
Enoplium.............. 38 I

fasciatum........... 386

humerale............. 38 I

leucophæum ... . . . . . . . 384

oculatum... . . . . . . . . 3 389

punctatissimum........ 395

quadrinotatum......... . 382

quadripunctatum......38 I, 382

thoracicum........... 395

venustum. ........... . 384

Entomostraca............. I72

Epinephelus drummond-hayi.... 53

falcatus............. 54

maculosus........... 52

morio.............. 53

striatus............ 53

Eques acuminatus.......... 60

Eremias spekii............. 409

Ericymba............... 275

buccata............ 275

Erimyzon.............. 25I

sucetta............ 25I

Erpobdella triannulata......... I 99

Erpobdellidæ................ 199

erythræum, Cichlasoma : . . . .99, I 24

erythræus, Heros. . . . . . . . . . I24

Erythrichthus............. I2 I

erythrogaster, Chrosomus. ..... 26I

Clerus.............. $35^{8}$

Esocidæ. . . . . . . . . . . . . 35, 295

Esox.................... 295

lucius. . . . . . . . . . . . 296

masquinongy . . . . . . . . . 297

vermiculatus........... 296

eta, Vivipara lanaoënsis var. . . . . 22 I

Etheostoma.............. 327

cœruleum............ 329

flabellare........... 330

iowæ.............. 328

jessix.............. 328

zonale............. 327

Eublepharidæ............ 3

Eucalia................. 302

inconstans........... 302

Eucinostomus californiensis...... I43

gula............... 60

harengulus............ 59

lefroyi.............. 60

pseudogula............ 60
PAGE.

Eudorina elegans. . . . . . . . . . . I 197

stagnale............... I97

Eulamia nicaraguensis.... . . . . 103

obscura............. 29

Eumycterias punctatissimus. . . . 148

Eupomacentrus............ 6I

adustus............ 6 I

chrysus............ 6I

fuscus.............. 62

leucostictus........... 62

Eupomotis.............. 314

gibbosus............313.315

euryorus, Lepomis........... $3 \mathbf{1 2}$

Eventognathi............. 247

evoluta, Terebra........... 2 I 7

excentricus, Ancylus......... 206

exile, Dorosoma. . . . . . . . . . . . I 55

exilis, Schilbeodes........... 246

Exocotida.............. 37

Exocotus bahiensis.......... 37

exiliens............. 37

furcatus............ 37

lineatus............ 37

rondeletii............ 37

rufipinnis........... 37

Exonautes exsiliens.......... 37

exiliens, Exocotus. ........... 37

exsiliens, Exonautes ......... 37

falcata, Mycteroperca......... 54

Seriola.............. 48

falcatus, Epinephelus......... 54

fasciata, Cregya.......386,389, 390

Gambusia............. I37

Molienisia............ I 38

fasciaculatum, Trogodendron . . . 366

fasciatum, Enoplium.... . . . . . 386

Pelonium............. $3^{87}$

fasciatus, Chilomeniscus....... I 4

Tetragonopterus.......... I34

fascifcra, Cymatodera........ 348

Faunas ater............. 217

Ficus.................. I68

Fierasfer bermudensis......... 85

Fierasferidæ............. 85

Fistularia tabacaria......... 38

Fistulariidæ.............. 38

flabellare, Etheostoma. . . . . . . 330

flabellicauda, Rivulus. . . . . . . . I 45 


\section{Field Museum of Natural History-Zoölogy, Vol. Vil.}

PAGE.

flavescens, Perca.......... 320

Sparisoma.......... 72

Trichodes.............. 370

Trichodes simulator var. . . 370

flavigularis, Gerrhosaurus. . . . . 409

flavolineatum, Hæmulon...... 57

flavovittatus, Mulloides....... 46

flavus, Noturus............ 244

fœtens, Synodus............ 34

formosus, Angelichthys....... 74

fragilis, Spingilla.......... 198

fredrichsthalii, Cichlasoma...... I 43

frontale, Cichlasoma......... 2 10

Phrynosoma........... I 12

frontalis, Placopterus........ 363

fuchsii, Cymatodera......... $35^{2}$

Fulica americana........... 195

fulvus, Bodianus.......... 52

Gobiesox............ I 49

punctatus, Bodianus...... 52

fumosa, Solenosteira.......... 217

Fundulus................ 299

bermudæ..........26, 35

diaphanus menona....... 299

dispar.............. 299

guatemalensis...... I36, I 80, I 84

notatus............. 300

pachycephalus.......... I36

punctatus........... I 36

funebris, Lycodontis......... 3 I

Furcaria cyanea........... 63

furcatus, Exocœetus.......... 37

furcifer, Paranthias.......... 55

fuscus, Eupomacentrus........ 62

fusiformis, Boleichthys........ 33 I

Gadidæ............. $33^{8}$

gahani, Hydnocera. . . . . . . . 379

gairdneri, Salmo. . . . . . . I 82, 293

galeata, Daphnia............ 204

Gallinule............ 195

Pelomedusa........... 4I4

Galeidæ...............29, I03

Gallinago delicata........... 195

Gallinule galeata........... I95

Gambusia affinis........... I55

annectens........... $\mathbf{I} 45$

fasciata............ I37
Gambusia affinis - Continued modesta............. I 38

nicaraguensis.......... I I3

terrabensis........... 146

gamma, Vivipara lanaoënsis var... 22 I

garnoti, Iridio.............. 68

Gasterosteidæ............ 302

Gempylidæ.............. 46

Gerres peruvianus........... I43

Gerrhonotus palmeri......... I2

scincicauda.......... I2

Gerrhosaurus flavigularis...... 409

major. ............4 409

Gerridæ............... 59

gibba, Pterophryne.......... 89

gibbosus, Eupomotis........313,315

Ginglymostomidæ.......... 29

Girardinus vandepolli......... 209

girodi, Thaneroclerus......363, 364

gladius, Regalecus.......... 86

Xiphias............. 46

glaucofrænum, Rhinogobius..... 8 8 I

glaucus, Trachinotus......... 49

glesne, Regalecus............ 86

Glossophonia lineata......... I99, 20 I

Glossophonidæ. ............ 199

Glyphidodon chrysurus....... 63

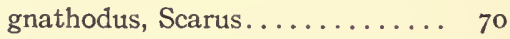

Gobiesocidæ.............. 149

Gobiesox fulvus............. I49

Gobiidæ...... 81, I3 I, I43, I49, I53

Gobiomorus gronovii......... 49

Gobius stigmaturus.......... 83

godmani, Rhamdia......... I34, I50

Goodea atripinnis......... I53, I56

goodei, Trachinotus.......... 49

Gopherus agassizii........... 3

gorhami, Hydnocera.......378, 379

gracilis, Pœcilochroa (Colyphus) . 363

graciosa, Uta.............. ro

graciosus, Sceloporus.......... I I

grahamiæ, Salvadora ......... I5

granadense, Cichlasoma........ I2 I

grandis, Cymatodera........354, 355

Pomadasis.............. I I6

granulosa, Cregya.......... 386

granulosum, Pelonium....... 386

griseus, Lutianus........... $5^{6}$

gronovii, Gobiomorus......... 49 
PAGE.

grossa, Cymatodera......... 355

grunniens, Aplodinotus........ 335

Haploidonotus. . . . . . . . I56

guacamaia, Pseudoscarus...... 69

guara, Caranx............ 47

guatemalensis, Amnicola...... 206

Chirostoma........... I I4

Fundulus......... I 36, I 80, I 84

Potamocarcinus......... 202

Rœboides..... 98, I I I, I 35, I 45

guentheri, Elapechis......... 405

gula, Eucinostomus.......... 60

gulosus, Chænobryttus........ 310

guttata, Promicrops......... $55^{2}$

guttatus, Percopsis........... 304

guttulatum, Cichlasoma... . . I I I , I 89

Gymnosarda alleterata. ....... 46

Gymnothorax punctatus....... 32

Gymnotidæ............. I35, I 84

Gymnotus carapo. . . . . . . I 135, I 84

gyrinus, Schilbeodes......... 245

haagi, Clerus................ $35^{8}$

Haagi, Pœcilochroa .......... 362

hæmatica, Hydnocera....377, 378, 379

Hæmulidæ.......... 57, 58, I I 6

Hæmulon bonariense......... 58

carbonarium.......... 57

flavolineatum......... 57

macrostomum......... $5^{8}$

melanurum.......... 57

sciurus............. 57

Hadropterus............. 32 I

aspro................ $32 \mathrm{I}$

Haploidonotus grunniens . . . . . . I 56

Haplomi . . . . . . . . . . . . . . . 295

hargreavesi, Papuina......... 216

Harpe rufa............... 63

harringtonensis, Atherina....... 40

Hartwegianus, Trichodes.... . . 373

hastingsi, Lutianus. . . . . . . . 57

hedricki, Cichlasoma......... I5 I

helioides, Teuthis........... 76

helleri, Crotalus............ I 17

Hemidactylus brookii... . . . . 406

mabouia ............ 406

Hemirhamphidæ.......... 36

Hemirhamphus brasiliensis..... 36

Hemirhombus aramaca....... 87
Hemirhombus aramaca-Cont'd. soleaformis........... 87

Hemisus marmorata........ . 404

hepatus, Teuthis.......... 76

harengulus, Eucinostomus...... 59

Herichthys. . . . . . . . . . . I I57

Heros balteatus. . . . . . . . . . . I 129

basilaris............. I22

centrarchus............ 126

citrinellus ............ I 2 I

dovii.............. I20

erythræus............ I 124

labiatus.............. I24

lobochilus............ I 25

longimanus........... 127

managuensis........... I I9

multispinosus.......... I3I

nicaraguensis........... 129

rostratus. . . . . . . . . . 126

Herotilpia................ I30

multispinosa.......... I3I

Heterandria lutzi............ I I37

pleurospilus............ I 37

heterodon, Notropis. . . . . . . . . 268

hians, Athlennes........... 36

hindii, Vipera............. 405

Hiodon. . . . . . . . . . . . . 286

alosoides............. 286

tergisus. . . . . . . . . . . . 286

Hiodontidæ... . . . . . . . . . . 285

Hippocampus antiquorum..... 40

brunneus............. 39

hippocampus.......... 40

hudsonius ............ 40

punctulatus........... 40

stylifer.............. 40

hippocampus, Hippocampus.... . 40

hippocastanea, Thias........ 2 I 7

hippurus, Coryphæna. ....... 49

hirundinella, Peridinium........ 197

hispidus, Monacanthus........ 78

Tropidurus.......... 4 16

histrio, Pterophryne.......... 89

hoeneli, Chamæleon ......... 4 413

Holacanthus tricolor......... 75

Holocentridæ............. 42

Holocentrus ascensionis. . . . . . 42

meeki............. 42

puncticulatus......... 43 
Holocentrus ascensionis - Cont'd. siccifer............. 43

tortugæ............. 43

hopei, Cymatodera............ 350

Hopfneri, Clerus ........... 36I

hoplomystax, Sparisoma...... 72

horni, Cymatodera.........349, 350

hotambœia, Leptodira ........ 406

hoyi, Argyrosomus.......... 292

hudsonius, Hippocampus....... 40

Notropis............ 27 I

Humboldtiana, Salix.......... I 68

humerale, Enoplium........ 38I

humeralis, Pyticera......... $38 \mathbf{I}$

Sardinella........... 34

humilis, Tetragonopterus..... 109, I 35

huronius, Argyrosomus........ 29I

Hybognathus............. 26I

nuchalis............. 26I

Hybopsis................ 277

æestivalis ........... 155

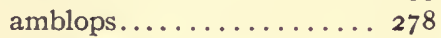

dissimilis........... 277

kentuckiensis......... 279

storerianus........... 278

Hydnocera aspera. . . . . . . . 379

discoidea........... 379

gahani............. 379

gorhami...............378, 379

hæmatica.............. 377 378, 379

nigro-ænea.......... 380

niveifascia........... 377

spinolæ................. 380

Hylambates bocagei. . . . . . . . 404

Hyperoartii.............. $23 \mathrm{I}$

Hypoplectrus puella......... 54

Hyporhamphus roberti........ 36

unifasciatus.......... 36

hystrix, Diodon........... 80

Ichthyomyzon.......... 23I

concolor............. 232

ictalops, Cottus............ 336

Ictalurus.............. 240

punctatus.......... 154, 240

Ictiobus..............249, 250

bubalus............. 249

urus.............. 249

Iguana rhinopla........... 192
Iguana rhinopla - Continued.

tuberculata.......... $4 \mathbf{I} 6$

Iguanidæ. . . . . . . . . 3, 192

illustris, Trichodes........369, 370

imberbis, Apogon............ 5I

incisor, Lepomis............ 31 3

inconstans, Eucalia........... 302

inermis, Dermatolepis........ 55

intermedius, Synodus........ 35

interrupta, Morone......... 333

iowæ, Etheostoma........... 328

irideus, Salmo............ I 82

Iridio................. 65

bivittatus.......... 63

cyanocephalus........ 68

decoratus............ 64

elegans............. 65

garnoti............. 68

meyeri..........65, 67

microstomus.......... 67

radiatus............. 68

isabella, Cypræa........... 217

isabellæ, Cymatodera......... 345

Isolemidia cariniceps........ 376

Isospondyli.............. 285

Isurus............... 30

jacksoni, Camæleon......... 4 $4^{\mathrm{I}} 3$

jamaicensis, Bithynis....... 202

jejunus, Notropis........... 270

jessiæ, Etheostoma......... 328

jonesi, Siphostoma..........26, 39

jonesii, Syngnathus.......... 39

jordani, Chirostoma. . . . . . . . . I 6

Lycodontis........... 32

Joturus pichardi........... I 48

kappa, Vivipara lanaoēnsis var. 22 I

kentuckiensis, Hybopsis........ 279

Kinixys belliana........... 414

kumlienii, Uranidea.......... 337

Kyphosidæ............. 60

Kyphosus sectatrix.......... 60

labiatum, Cichlasoma.......99, 124

labiatus, Heros............. I 24

Labidesthes.............. 305

sicculus............ 305

labofusca, Leptodira......... 415 
PAGE.

Labridæ..................... $6_{3}$

Labrisomus lentiginosus....... 83 nuchipinnis.......... 84

Lachnolaimus maximus....... 63

Lactophrys tricornis......... 79 trigonus............ 79

triqueter............ 79

lacustris, Ameiurus......... 242

lævicollis, Cymatodera. . . . . . 345

Lagodon rhomboides......... 59

lalandi, Seriola............ 48

lamia, Carcharhinus......... 29

Lamnidæ............... 30

Lampetra.............. 232

wilderi........... 232

Lampropeltis boylii.......... 15

zonata........... 15

Lampyris pilosa........... $3^{83}$

lanaoënsis, Vivipara..........22 I

largillierti, Sphæromelania . . . . 205

laticinctus, Clerus......... 362

latus, Caranx............. 47

Lebasiella. . . . . . . . . . . . 397

marginella.......... 397

nigripennis. . . . . . . . . 397

pallipes............. 397

lecontei, Clerus. . . . . . . . . . 359

Priocera................ 356

lefroyi, Eucinostomus......... 60

Ulæma.............. 60

Lefroyia bermudensis......... 85

lemniscatus, Cnemidophorus.... . 4I7

lentiginosus, Labrisomus. . . . . . 83

lepidus, Crotalus........... I9

Lepisosteidæ . . . . . . . 104, 153, 237

Lepisosteus............. 104, 237

osseus............ 153, 237

platostomus........... 238

tristoechus.......... ro5

tropicus............. I04

Lepomis................ 3 I I

cyanellus............ 3 I I

euryorus........... $33^{12}$

incisor............. $3^{1} 3$

megalotis.......... 5 56, 3 r 3

pallidus........... 156, I 75

Leptecheneis naucrates........ 83

Leptocephalidæ............ 3 I

Leptocephalus............. 30
Leptodactylus microtis......... 192

Leptodira albofusca.......... 415

Leptodira hotambœia ........ 406

Leptopoma callichloros......... 216

pumilum...........216

Leptops................ 244

olivaris............. 244

Leuciscus nigrescens.......153, 154

leucophæa, Cregya . . 384, 385, 386, 388

(vetusta), Cregya........ 390

leucophæum, Pelonium....... 384

leucostictus, Eupomacentrus.... 62

leucurus, Spermophilus......... I5

libertatis, Opisthonema........ II 2

limantouri, Pœcilia ........... I 38

limi, Umbra. . . . . . . . . . . . 297

Limnæidæ.... . . . . . . . . . 206

lineata, Glossiphonia. . . . . . 199, 201

lineatus, Anolis............ 416

Exocœtus............ 37

lineolatum, Pelonium. . . . . 389, 390

Liognathidæ.............. 143

lobochilus, Cichlasoma....... 124, 125

Heros............. 125

Lobotes surinamensis........ 55

Lobotidæ............... 55

longiceps, Eleotris.......... I 3 I

Philypnus........... 13 I

longicornis, Cymatodera....... 343

longimanus, Cichlasoma....... 153

Heros............. 127

longispina, Daphnia......... 204

Lota.................................. 338

maculosa............... $33^{8}$

lucayanum, Asymmetron...... 29

lucens, Potamogeton.......... $17 \mathrm{r}$

lucifer, Crotalus............ I7

lucius, Esox. . . . . . . . . . . 296

lunatus, Clerus............. 359

Platophrys.......... 86

Lutianidæ............. 55, 140

Lutianus apodus.......... 55

aya............ 57

buccanella.......... 57

griseus............ $5^{6}$

hastingsi ......... 57

synagris............ 56

vivanus............. 56

lutrensis, Notropis........... 155 
432 Field Museum of Natural History-Zoölogy, Vol. ViI.

PAGE.

lutzi, Heterandria.......... I37

Lycodontis funebris., .......... $3 \mathrm{I}$

jordani............. 32

moringa............ 32

ocellatus........... 32

sanctæ-helenæ......... 32

Lycophidium capense......... 405

Lygodactylus picturatus....... 406

Lygosoma sundevallii.......... $4^{12}$

Lynceidæ................ 204

lynx, Cypræa............ 217

mabouia, Hemidactylus...... 406

Mabuia brevicollis........... 4Io

megalura........... 4II

quinquetæniata......... 4 Io

striata............. 4 II

varia............. 4 II

mackayi, Siphostoma........ 38

macracarithum, Cichlasoma..... I 40

macrophthalmus, Sardinella.... 34

Macrorhamphosidæ........... $3^{8}$

Macrorhamphosus scolopax...... 38

macrostomum, Hæmulon....... . $5^{8}$

Macroteleia.............. 339

Macrotelus........... 339, 340

cinctipennis.......... 34I

terminatus.......... $34^{2}$

terminatus var. pallipes.... 34 I

macularius, Acititis........... I95

maculatus, Apogon.......... $5^{\text {I }}$

Aulostomus.......... 37

Canthidermis.......... 76

Dormitator............ r 43

Philypnus............ I 43

Upeneus . . . . . . . . . . 45

maculicauda, Cichlasoma..... I4I, I5 I maculicolle, Pelonium........ 388 maculicollis, Cregya. . . 385, 388, 390 maculosa, Lota............ 338 maculosus, Epinephelus....... 52 magister, Sceloporus.......... Io major, Gerrhosaurus......... 409 Malacanthidæ........... 83

Malacanthus plumieri........ 83 mammilla, Polinices......... 2 r 8 managuensis, Cichlasoma $119,143,152$

Heros.............. 119

Pimelodus............ 105 managuensis, Cichlasoma-Cont'd

Rhamdia............ 105

manana, Cichlasoma.......... I4I

Mapo soporator............81, 83

margaritaceus, Turbo........ 2 I 8

marginella, Lebasiela. . . . . . . . 397

marginellus, Corynetes. . . . . . 397

Necrobia. . . . . . . . . . 397

marginipenne, Pelonium....... 389

marinus, Bufo........... r91

marmorata, Clemmys........ 3

Hemisus. . . . . ..... 404

Rappia............. 404

marmoratus, Symbranchus..... I5I

Marsipobranchii..........227,231

martinicus, Upeneus. . . . . . . . 46

mascariensis, Rana......... 403

masquinongy, Esox.......... 297

maximus, Lachnolaimus........ 63

mearnsi, Uta.............. 9

mearnsii, Vivipera.......... 221

meeki, Holocentrus. . . . . . . . . 42

Thyrina............ r 39

Megalops................ II I

megalotis, Lepomis......... 156,313

megalura, Mabuia............ 4I I

megatoma, Elasmocerus....... 342

Monophylla.......... $34^{2}$

Melanidæ............... 205

Melaniris............... I I 4

balsanus........... r $\mathbf{I}_{4}$

sardina............99, II4

melanops, Minytrema........ 252

melanurum, Hæmulon....... 57

melas, Ameiurus. . . . . . . . . . 243

mendana Papuinia........... 215

Menidia menidia........... 4I

notata........... 4 I

menidia, Menidia.......... 4 I

menona, Fundulus diaphanus. . . . 299

mentovarius, Zamensis....... 4r 5

meta, Papuina............ 215

mexicana, Pœcilia........... 1,38

mexicanus, Astyanax. . . . . . 153, I 55

meyeri, Iridio........... 65, 67

microlepis, Mycteroperca...... 54

Microperca............. 33 I

punctulata.......... 332

microporum, Colastrum ....... 196 
Micropterus................. 3 I 5

dolomieu.............. 316

salmoides.......... I75, 316

micropthalmus, Astyanax...... I 84

Tetragonopterus........ I35

microscutata, Uta.......... I0

Microspathodon chrysurus..... $62^{\circ}$

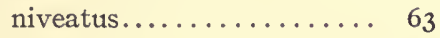

microstoma, Dina.......... 200

microstomus, Carpiodes........ I54

Iridio.............. 67

microtis, Leptodactylus. . . . . . . I I92

micrurum, Syacium......... 87

militare, Pelonium.......... 38I

Pyticera............. 38I

milleri, Cichlasoma.......... I42

minutus, Arthroleptis....... 403

Minytrema.............. 252

melanops........... 252

mirabilis, Phenacobius. ...... 276

mitchellii, Crotalus.......... I 8

miurus, Schilbeodes... . . . . . . . 247

mixta, Cregya.........390, 391, 392

modesta, Gambusia.......... 138

mojarra, Cichlasoma........ I40, I4I

Mola mola.............. 80

mola, Orthagoriscus.......... 80

Molidæ.................. 80

Molienisia fasciata........... 138

Monacanthidæ............ 76

Monacanthus ciliatus........ 77

hispidus.................... $7^{8}$

tuckeri.............. 78

monilis, Thanasimus........ 362

Monophylla........... 339, 340

californica......3 340,341, 342

cinctipennis..........340, 34 I

megatoma........... 342

substriata......3 340, 341, 342

terminata..........34, 342

monticola, Agonostomus . . . . 139, I 47

Aulicus........... 364, 365

Thanasimus.......... 357

moringa, Lycodontis.......... 32

morio, Epinephelus.......... 53

Morone................. 333

interrupta........... 333

morus, Cerithium.......... 217

motaguense, Cichlasoma. I 42, I 51, I 74 mowbrayi, Rhinogobius....... 8I

Moxostoma............... 253

anisurum............ 254

aureolum............ 253

breviceps............. 254

congestum............ I 54

Mugil brasiliensis........... 4 I

curema............. 4I

trichodon............ 4 I

Mugilidæ..........41, I39, I47

Mullidæ................. 45

Mulloides flavovittatus........ 46

multispinosa, Herotilpia........ I31

multispinosus, Heros... . . . . . . I 3 I

Murænidæ.............. 3I

murinus, Cnemidophorus....... 4I 7

Mustelus canis.............. 29

mutillarius, Clerus.... . . . . . . 357

Mycteroperca bonaci......... 53

bowersi............. 54

falcata............. 54

microlepis........... 54

tigris.............. 54

venenoso apta.......... 54

Myliobatidæ.............. 30

myops, Trachinocephalus...... 35

Myrichthys acuminatus........ 3I

Myridæ................ 3I

Myrophis punctatus......... 3I

namaycush, Cristivomer........ 294

Nanina nitidissima.......... 2 I5

narinari, Aëtobatus......... 30

nasuta, Rhamdia........... 207

nasutus, Astyanax. . . . . . . . . I08

natalensis, Phrynobatrachus. . . . 403

natalis, Ameiurus. ... . . . . . . . 242

natans, Salvinia............ I70

Natica tessellata........... 218

Naucrates ductor............. 48

naucrates, Echeneis.......... 83

Leptecheneis........... 83

nebulosus, Ameiurus. . . . . . . . . 242

Necrobia marginellus. . . . . . . . 397

Neetroplus.......... I30, I 56, I 57

carpintus............ I 56, I 57

nematopus....... I 30, I 53, I 57

nicaraguensis........... I30

neglectus, Chlorophis........ 406 
PAGE.

Nematognathi............. 239

Neomænis argentiventris....... 140

Neotoma................ ${ }^{15}$

Nerita aurantia........... 2 I 8

signata............ 218

strigillata........... 218

versicolor............ 218

Neritina cornea........... 218

petiti.............. 2 I 8

pulligera............ 219

nero, Aulicus............364, 365

nexus, Trichodes............ $37 \mathrm{I}$

nicaraguense, Cichlasoma.... I I29, I30

Rhamdia............ I 34

nicaraguensis, Carcharhinus..... 103

Eulamia............. 103

Gambusia........... I I 3

Heros.............. I29

Neetroplus........... $\mathbf{1}_{30}$

Paragambusia........... I I 3

Pimelodus............. 106

Rhamdia............. I06

nigrescens, Centropomus. . . . I40, I 74

Leuciscus........... I53, I 54

nigricans, Catostomus......... 257

nigricolor, Cnemidophorus...... 417

nigripennis, Argyrosomus. . . . . 293

Lebasiella. . . . . . . . . 397

Orthopleura.......... 395

nigritum, Cichlasoma........ 128

nigriventris, Clerus........ 359

nigro-ænea, Hydnocera....... 380

nigrofasciatum, Cichlasoma. . 141, 180,

. ............... 189

nigrum, Boleosoma.......... 324

niloticus, Varanus.......... 409

nitidissima, Nanina.......... 2 I5

nitidus, Adelphoclerus....... 357

Chlorichthys.......... 68

niveatus, Microspathodon...... 63

niveifascia, Hydnocera....... 376

notata, Menidia........... $4 \mathrm{I}$

Uma................ 3

obcordata, Pistia........... I 70

obscura, Eulamia........... 29

obtusirostris, Bosmina........ 204

occipitalis, Chioractis........ I5

ocellatus, Lycodontis........ 32 ocellatus, Lycodontis - Cont'd.

Platophrys........... 86

oculata, Cregya.....386, 389, 390,39r

oculatum, Enoplium......... 389

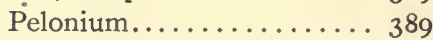

oculatus, Clerus............ 389

Ocyurus chrysurus.......... 57

œrstedii, Astyanax.......... ${ }_{4} 45$

Ogilbia cayorum.......... 85

oglinum, Opisthonema........ 33

oithnoides, Cyclops........... 205

olivaris, Leptops. . . . . . . . . . 244

onusta, Chariessa pilosa var..... 394

Ophichthyidæ............ 3I

Ophichthys triserialis........ 3I

opifex, Clerus............. 360

Opisthonema libertatis........ 112

oglinum.............. 33

Opsopœodus............... 263

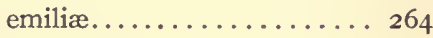

orcutti, Sceloporus........... x I

Orcynus alliteratus.......... 46

thynnus............ 46

oresterus, Trichodes.......368, 369

ornaticollis, Cleronomous....... 362

ornatus, Clerus decussatus var... 36r

Trichodes....372, 373, 374, 375

Orthagoriscus mola......... 80

Orthopleura............. 393

bimaculata.................... 395

damicornis.........394, 395

nigripennis................ 395

punctatissima...................... 395

quadraticollis.......... 393

texana............394, 395

Orthopristis chrysopterus....... 58

oryhynchus, Rhamphiophia..... 405

osseus, Lepisosteus. . . . . . . 153, 237

Ostraciidæ............... 79

Oxybelis acuminatus......... 4 $4^{16}$

Oxyechus vociferus.......... 196

pachycephalus, Fundulus....... I 36

pagodula, Vivipara.......... 221

Palæmondiæ............... 202

pallida, Cymatodera.......... 344

pallidus, Lepomis.......... 156, 175

pallipes, Corynetes.......... 397

Lebasiella........... 396 
pallipes, Corynetes - Continued.

Macrotelus terminatus var.. 34 palmeri, Gerrhonotus........ I2

Paludicola brachyops........ 4 4 5

palustris, Potamides........ 2 I 7

pancheri, Placostylus........ 2 rg

Panicum.............. I 70

papillosum, Syacium........ 87

Papuina............... 2 I 6

adonis............ 215

hargreavesi.......... 2 I6

mendana............ 215

$\operatorname{meta...\ldots \ldots \ldots \ldots \ldots ...........~} 215$

xanthochila.......... 2 I5

yulensis............. $2 \mathrm{I} 6$

Paragambusia.............. Ir 3

nicaraguensis.......... I I 3

Paranthias furcifer......... 55

Parapetenia.............. I 19

parryi, Rhamdia............ I34

partelloi, Vivipara............22 I

pelagicum, Siphostoma......... 39

pellucida, Ammocrypta . . . . . . 326

pellucidus, Psenes... . . . . . . . . 49

Pelomedusa galeata.......... 4 I 4

Pelonium................ 383

bilineicolle... . . . . . . 392

fasciatum........... 387

granulosum........... 386

leucophæum........... 384

lineolatum... . . . . . 389, 390

maculicolle........... 388

marginipenne......... 389

militare............ 38r

mixtum............ 39r

oculatum............ . 389

pennsylvanicum........ 395

quadrinotatum.... . . . . 392

vetustum............. 384

peninsularis, Cymatodera...... 346

Trichodes......... 368, 369

pennsylvanicum, Pelonium. . . . 395

Perca................ 320

flavescens........... 320

Percidæ............... 31 7

Percina............... 320

caprodes............ 320

Percopsidæ............... 303

Percopsis................ 303
Percopsis-Continued.

PAGE.

guttatus.............. 304

Peridinidæ............... 197

Peridinium hirundinella........ 197

tabulatum............ 197

Perodipus............... 9

peruvianus, Gerres........... I 443

petenensis, Pœcilia........... r38

petensis, Amnicola...... . . . . . 206

petiti, Neritina............ 2 18

Petrometopon cruentatus...... 54

Petromyzonidæ............231

Phenacobius............. 276

mirabilis........... 276

Philypnus............... r 3 I

dormitor......... r3 r, I 53, r 74

longiceps............. I3I

maculatus............. 143

phœbe, Prionodes........ . . . . . 54

Phrynobatrachus natalensis.... . 403

Phrynomantis bifasciata....... 404

Phrynosoma blainvillii........ I2

coronatum........... I2

frontale............ I2

platyrhinus........... 12

Phyllopoda............... 203

pichardi, Joturus. . . . . . . . . . I I 48

picta, Pterophryne. . . . . . . . . . 89

picturatus, Lygodactylus. . . . . . . 406

picudilla, Sphyræna . . . . . . . . 42

pigmentarius, Apogon......... 50

pilosa, Chariessa . . . . . . . . . 383, 394

Pimelodus managuensis. . . . . . . 105

nicaraguensis.......... 106

rogersi................ I44

Pimephales. . . . . . . . . . . 262, 266

notatus........... I54, 263

promelas............. 262

pipiens, Rana.............. I9I

Pisces... . . . . . . . . . . 227, 234

Pistia obcordata........... I 70

Pityophis catenifer............ I5

Placopharynx duquesnii. . . . . . . 248

Placopterus............... 363

frontalis............. 363

rufipes.............. 363

varia.............. 363

Placostylus............... 2 r 9

dorseyi............216,219 


\section{Field Museum of Natural History-Zoölogy, Vol. ViI.}

\section{PAGE.}

Placostylus - Continued.

(Leucocharis) dorseyi..... 219

pancheri........... 2 I9

pancheri var. candida..... 2I9

Planaxis sulcatus.......... 217

Planorbis subpronus......... 206

Platophrys lunatus.......... 86

ocellatus............ 86

platostomus, Lepisosteus....... 238

Platynoptera............. 38 I

platyodon, Carcharhinus....... 29

Platypœcilus tropicus......... I46

platyrhinus, Phrynosoma....... I2

Plectrypops retrospinis........ 45

Pleuronectidæ............ 86

pleurospilus, Heterandria....... I37

plumbea, Pœcilochroa......... 363

plumieri, Malacanthus........ 83

Scorpæna........... 80

Podicipedidæ............. I94

Pœcilia................. I 13

butleri............. I 38

caudata............. 209

chisoyensis........... I38

dovii............. I $3, \mathrm{I}_{3} 8$

limantouri........... I38

mexicana............ 138

petenensis........... I 38

salvatoris........... I 38

sphenops 98, I I 3, I 37, I67, I 80, I 86

tenuis............. I 47

thermalis............ $\mathbf{I}_{3} 8$

Pœciliidæ. . .35, I I 2, I 36, I 45, I 5 I, I 55, $\ldots \ldots \ldots \ldots \ldots \ldots$ I 84,298

Poecilochroa...........358, 363

cyanipennis.......... 362

(Colyphus) gracilis...... 363

Haagi............... 362

plumbea.............. $3^{63}$

Polinices mammilla.......... 218

sebæ.............. 218

Polyodon................ 234

spathula........... 235

Polyodontidæ............ 234

Pomacentridæ............. 60

Pomadasis................ I I6 grandis............. I I6

Pomolobus............... 288 chrysochloris.......... 288
PAGE.

Pomoxis................ 307

annularis............. 308

sparoides.......... I75, 308

Pontinus............... 26

Pontius.............. 8

Porzana carolina............ 195

Potamides palustris.......... 2 I 7

Potamocarcinus guatemalensis... 202

Potamogeton........ I 7 I, I 80, I86

lucens.............. I7 I

Potamonidæ............. 202

Priacanthidæ.............. 55

Priacanthus arenatus........ 55

cruentatus........... 55

principis, Antennarius......... 89

Pterophryne.......... 89

Priocera................ 356

albomaculata........... 384

castanea.............. 356

lecontei............ 356

Prionodera............... 396

tantilla............. 396

Prionodes phøbe............ 54

Pristidæ................ I04

Pristis................ I04

antiquorum........... 104

prognathus, Argyrosomus. . . . . 292

promelas, Pimephales........ 262

prometheus, Promethichthys.... 46

Thyrsites............ 46

Promethichthys prometheus.... 46

Promicrops guttata......... 52

Psammophis sibilans.......... 405

Psenes pellucidus............ 49

Pseudoclerops............. 357

pseudogula, Eucinostomus...... 60

Pseudoscarus acutus......... 70

guacamaia........... 69

psittacus............ 70

quadrispinosus......... 70

Pseudoxiphophorus bimaculatus.. I5I psittacus, Pseudoscarus....... 70

Pterophryne gibba.......... 89

histrio............ 89

picta............... 89

principis........... 89

puella, Hypoplectrus.......... 54

pulchra, Anniella............ I3

pulex, Daphnia............. 204 
pulligera, Neritina........... 2 r 9

pumilum, Leptopoma......... 216

punctatissima, Orthopleura..... 395

punctatissimum, Enoplium..... 395

punctatissimus, Eumycterias..... I 48

punctatum, Cichlasoma........ 2 I0

punctatus, Decapterus......... 48

Fundulus............ 136

Gymnothorax................. 32

Ictalurus.......... I54, 240

Myrophis............. 3I

puncticulatus, Holocentrus..... 43

punctulata, Microperca....... 332

punctulatus, Hippocampus..... 40

pungitius, Pygosteus........... 303

Pygosteus.................. 303

pungitius.............. 303

pyramidata, Pythia........... 2 I6

Pythia pyramidata $\ldots \ldots \ldots \ldots 2 \mathrm{~m} 6$

Pyticera.......................... $38 \mathrm{r}$

humeralis........... $3^{8} \mathbf{I}$

militare............... $38 \mathbf{I}$

quadripunctata........ 382

quadraticollis, Orthopleura. . . . 393

quadrilateralis, Coregonus...... 290

quadrinotata, Cregya........ 393

quadrinotatum, Enoplium...... 382

Pelonium............ 392

quadripunctata, Pyticera...... 382

quadripunctatum, Enoplium. 381,382

quadrisignata, Cregya....390, 392, 393

quadrisignatum, Pelonium..... 392

quadrisignatus, Clerus . . . . . 359, 362

quadrispinosus, Pseudoscarus.... 70

quercinus, Chloritis........2 16, 220

Querquedula discors.......... I94

quinquetæniata, Mabuia...... 4I0

Rachycentridæ............ 49

Rachycentron canadus........ 49

radians, Rhus............. 385

Sparisoma.......... 72

radiatus, Iridio............ 68

radiosus, Antennarius........ 89

raii, Brama.............. 50

Rallidæ................ I95

ramicornis, Chariessa........ 383

Rana mascariensis.......... 403

pipiens........... I9I
Ranidæ................ I9I

Ranzania truncata........... 80

raphidoma, Tylosurus......... 36

rapicaudus, Thecadactylus...... 416

Rappia cinctiventris........ 404

marmorata........... 404

Regalecidæ............... 86

Regalecus gladius........... 86

glesne............... 86

regani, Astyanax........... 207

Rhamdia........... I 44

regularis, Bufo........... 404

Remora remora........... 83

remora, Remora............ 83

Reptilia........................ 415

resimus, Causus........... 405

reticulatus, Chilomycterus...... 80

retrospinis, Plectrypops....... 45

Rhamdia............... I05

barbata............. I06

brachycephalus. . . . . . . I44

cabreræ........93, 134, I 83

godmani.......... I34, 150

managuensis........... I05

nasuta............. 207

nicaraguense.......... 134

nicaraguensis.......... I06

parryi.............. I34

regani............... I44

Rhamphiophis oxyrhynchus.... 405

Rhinichthys........... 28r

atronasus............28

cataractæ...........281

Rhinogobius glaucofrænum..... 8 $8 \mathrm{r}$

mowbrayi............ $8 \mathrm{I}$

rhinopla, Iguana........... I92

Rhomboganoidea........... 236

rhomboides, Lagodon......... 59

Rhomboplites aurorubens...... 26

Rhus radians.............. 385

Rhypticus bistrispinus........ 55

ricei, Cottus............... 336

ricinus, Drupa............ 217

rigaudi, Ceriodaphnia........ 204

rimator, Bathystoma........ $5^{8}$

riverendi, Cyprinus........... 208

Rivulus............... I 45

flabellicauda.......... I45

roberti, Hyporhamphus...... 36 
PAGE.
PAG. . . . . . . . . . . . . . . . . .

Roccus................ 333

chrysops............ 333

rochei, Auxis.............. 46

Rœboides. . . . . . . . . . . . . I I I

guatemalensis...98, I I I, I35, 145

rogersi, Pimelodus. .......... I44

rondeletii, Exocœetus......... 37

rosipes, Novaculicthys.... . . . . 69

rostrata, Anguilla... . . . . . . . 285

rostratum, Cichlasoma... I26, I 28, I52

rostratus, Canthigaster.......26, 80

Heros.............. 126

ruber, Bodianus fulvus. . . . . . . 52

Caranx.............. $4^{8}$

Crotalus............. I;

rubicundus, Acipenser......... 236

rubrifrons, Notropis... . . . . . . 274

rubriventris, Thanasimus...... 357

rufa, Harpe............... 63

rufipes, Placopterus.......... 363

rufipinnis, Exocœtus. . . . . . . . 37

rufiventris, Clerus. . . . . . . . 358

rupestris, Ambloplites... . . . . I75, 309

Salariichthys textilis......... 85

Salix Humboldtiana........... I68

Salmo................... 293

gairdneri........... I 82, 293

irideus............. I 182

sebego.............. I 82

salmoides, Micropterus. . . . . I I75, 3 I 6

Salmonidæ. . . . . . . . . . . . 289

Salvadora grahamiæ.......... I5

salvatoris, Pœcilia ........... 138

salvini, Clerus. . . . . . . . . . 36 I

Salvinia natans. . . . . . . . . . I 70

sanctæ-helenæ, Lycodontis..... 32

santarosæ, Cymatodera...... . 35I

santarosaliæ, Notropis. . . . . . . . I I55

sardina, Melaniris.........99, I I4

Sardinella anchovia......... 34

humeralis............ 34

macrophthalmus........ 34

Sargassum........48, 49, 55, 62, 89

Sauromalus ater........... 9

saurus, Synodus............. 35

sayanus, Aphredoderus........ 306

scaber, Antennarius.......... 87

Scaridæ................ 69
PAGE.

Scarus acutus............ 70

cœruleus.............. 69

croicensis............. 69

gnathodus............ 70

tæniopterus........... 70

trispinosus.......... 70

vetula............. 70

Sceloporus biseriatus.......... I I

clarkii.............. I I

graciosus............ I I

magister............. Io

orcutti............ I I

zosteromus........... I I

Schilbeodes............... 245

exilis............... 246

gyrinus............. 245

miurus.............. 247

schoepffi, Alutera.......... 76

Sciænidæ.......... . 60, I56, 334

scincicauda, Gerrhonotus....... 12

Scirpus................ 170

sciurus, Hæmulon. . . . . . . . . 57

scobinata, Tellina........... 2 I9

Scolopacidæ............... I95

scolopax, Centriscus......... 38

Macrorhamphosus........ $3^{8}$

Scombridæ.............. $4^{6}$

scombrinus, Decapterus........ $4^{8}$

Scorpæna agassizii........... 80

plumieri............. 80

Scorpænidæ.............. 80

scripta, Alutera............ 77

scriptus, Alutarius.......... 77

sebæ, Polinices.............. 218

sebego, Salmo............... I 82

sectatrix, Kyphosus........... 60

Selachostomi.............. 234

sellicauda, Apogon........... 5I

semiornata, Coronella........ 406

Semotilus. . . . . . . . . . . . . 280

atromaculatus......... 280

senegalensis, Cassina......... 404

septentrionalis, Ceryle americana 196

serica, Verticaria........... I4

Seriola falcata............. 48

lalandi............. 48

zonata............. 48

Serranidæ. . . . . . . . . . 52, 332

shumardi, Cottogaster. . . . . 322 
sibilans, Psammophis ........ 405

siccifer, Holocentrus......... 43

sicculus, Labidesthes......... 305

Sicydium altum........... I 49

Sididæ. . . . ........... 203

signata, Nerita........... 218

Siluridæ. . .93, ro5, I 34, I 44, I 50, I 54, $\ldots \ldots \ldots \ldots \ldots \ldots \ldots \ldots \ldots$

silus, Crotaphytus........... 9 simulator, Trichodes.......370, 37 I

Siphostoma dendriticum....... 39 elucens.............. 38 jonesi...............26, 39 mackayi............. 38 pelagicum........... 39

snowi, Cymatodera... . . . . . . . 349 soleæformis, Hemirhombus. . . . . 87 Solenosteira fumosa.......... 2 r 7 soporator, Mapo..........81,83 soror, Cymatodera..... . . . . . 342 Sparidæ................. 59 Sparisoma abildgaardi....... 7 r aurofrenatum......... 72 flavescens........... 72 hoplomystax......... 72 radians.............. 72 viride............. 72 xystrodon............ 73 sparoides, Pomoxis........ 175, 308 spathula, Polyodon.... . . . . . . 235 spekii, Eremias. ... . . . . . . . . . 409 spengleri, Spheroides... . . . . . . 79 Spermophilus leucurus......... I5 Sphæromelania largillierti...... 205 Sphagebranchus anguiformis.... . 3I sphegeus, Clerus. . . . . . . . . $35^{8}$ sphenops, Pœcilia.98, I 1 3, I 37, 167, I80,

Spheroides

Spheroides spengleri.......... 79

Sphyræna barracuda......... 4I

borealis........... 42

picudilla........... 42

sphyræna........... 42

sphyræna, Sphyræna......... 42

Sphyrænidæ............. 4I

Sphyrna zygæna........... 30

Sphyrnidæ............... 30

spilurum, Cichlasoma......... I5 I

spinolæ, Hydnocera........... 380
PAGE.

Spongilidæ................ I98

Spongilla fragilis.............. 198

stagnale, Eudorina........... 197

stansburiana, Uta........... Io

stejnegeri, Cnemidophorus...... I I

stellifer, Antennarius.......... 89

stigmaturus, Gobius.......... 83

Stizostedion. ............. 318

canadense............ 318

vitreum ............. 319

Stolephorus chœerostomus....... 34

storerianus, Hybopsis......... 278

striata, Mabuia........... 4r I

striatum, Bathystoma........ $5^{8}$

striatus, Chætodon.......... 74

Epinephelus.......... 53

strigillata, Neritina......... 2 I 8

Stromateidæ............... 49

Strombus campbelli.......... 217

stylifer, Hippocampus......... 40

subpromus, Planorbis......... 206

subsimilis, Cymatodera. . . . . . 343

substriata, Monophylla...340, 341, 342

sucetta, Erimyzon.......... 25I

Sueciæ, Cladocera ... . . . . . . . . . 204

sulcatus, Planaxis.......... 217

sundevallii, Lygosoma. . . . . . . 412

surinamensis, Ameiva....... 417

Lobotes.... . . . . . . . . . 55

Syacium micrurum......... 87

papillosum........... 87

Symbranchidæ............ I5I

Symbranchus marmoratus...... I5I

synagris, Lutianus........... $5^{6}$

Syngnathidæ.............. 38

Syngnathus jonesii.......... 39

Synodontidæ............. 34

Synodus fœtens............. 34

intermedius.......... 35

saurus.............. 35

Systenoderes dastoides. . . . . . 362

tabacaria, Fistularia......... $3^{8}$

tabulata, Testudo.......... 418

tabulatum, Peridinium........ 197

tæniopterus, Scarus.......... 70

tantilla, Prionodera.......... 396

tantillus, Clerus (Thaneroclerus). 396

Tarpon atlanticus..........33, I I I 


\section{Field Museum of Natural History-Zoölogy, Vol. Vit.}

PAGE.
PA. . . . . . .

Tectarius bullatus..........2 18

Teiidæ . . . . . . . . . . . 13, I93

Tellina scobinata........... 2 I9

tenellus, Trichodes. . . . . . . . 373

Trichodes ornatus var..... 373

tenue, Cichlasoma........... 94

tenuis, Cynodonichthys........ I45

Pocilia.............. I 47

Terebra evoluta............ 217

tergisus, Hiodon............ 286

terminata, Monophylla..... .341, 342

terminatus, Elasmocerus. . . . . . 342

Macrotelus........... 342

Tillus.............. 342

terrabensis, Gambusia . . . . . . . I I46

terrificus, Crotalus.......... 416

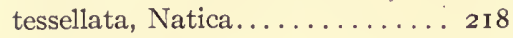

Testudinidæ.............. 3

Testudo tabulata........... 4I 8

Tetragonopterus æneus . . . . . . . I 135

fasciatus............. 134

humilis............. I09, 135

micropthalmus.......... 135

Tetraodontidæ ...........79, 148

Teuthididæ............. 75

Teuthis bahianus........... 75

cœruleus............. 75

helioides............. 76

hepatus............. 76

texana, Chariessa.......... 382

Orthopleura. . . . . . 394, 395

textilis, Salariichthys......... 85

Thanasimus............. 359

dubius............. 357

monilis............ 362

monticola........... 357

rubriventris.......... 357

Thaneroclerus............. 396

girodi............. 363, 364

sanguineus. . . . . . . . . . 364

thazard, Auxis............. 46

Thecadactylus rapicaudus. . . . 4 416

thermalis, Pœecilia............ 138

Thias hippocastanea......... 217

thoracicum, Enoplium. . . . . . . 395

thoracicus, Clerus. . . . . . . 358, 362

Thorichthys............... I 26

tigris, Cnemidophorus......... I3

Crotalus............. I6
Tigris-Continued.

Mycteroperca.......... 54

Tillus damicornis... . . . . . . . 395

terminatus.......... 342

Thunnus thynnus.......... 46

thynnus, Orcynus........... 46

Thunnus............ 46

Thyrina meeki............. I 39

Thyrsites prometheus........ 46

torosa, Cymatodera.......... 347

tortugæ, Holocentrus. . . . . . . . 43

Trachinocephalus myops....... 35

Trachinotus glaucus......... 49

goodei............... 49

Trachurops crumenophthalmus.. $\quad 48$ transandeanus, Chonophorus..... I 50 triannulata, Erpobdella. . . . . . . I I99 Trichodes.............366, 370

apivorus......3.370, 37 I, 375 apivorus var. interruptus. . 376 bibalteatus..........370, 37 I bimaculatus. . . . . . . . . 374 bisignatus............ 374

Douglassianus......... 373

flavescens........... 370

Hartwegianus......... 373

illustris. . . . . . . . . 369, 370

nexus............. 372

nuttalli.......373, 374, 375

oresterus.......... 368, 369

ornatus.....372, 373, 374, 375

ornatus var. tenellus... . . 373

peninsularis........ 368, 369

simulator...........370, 37 I

simulator var. flavescens. . 370

tenellus............. 373

trichodon, Mugil........... 4 I

tricolor, Holacanthus. . . . . . . . 74

tricornis, Lactophrys... . . . . . . . 79

trigonus, Lactophrys... . . . . . . . 79

trimaculatum, Cichlasoma...93, 94, 95 , $\ldots \ldots \ldots \ldots$ I $40, \mathbf{I}_{74}$

Trimerorhinus tritæniatus . . . . . 405 triqueter, Lactophrys. . . . . . . . 79 triserialis, Ophichthys........ 3 I trispinosus, Scarus........... 70 tristoechus, Lepisosteus......... 105 tritæniatus, Trimerorhinus .... 405 Trogodendron............ 366 
Trogodendron-Continued.

Edwardsii........... 366

fasciaculatum......... 366

tropicus, Atractosteus......... 104

Lepisosteus.......... I04

Platypœecilus .......... $\mathbf{1 4 6}$

Tropidurus hispidus......... 4 I6

truncata, Ranzania.......... 80

tuberculata, Iguana.......... $4 \mathbf{1 6}$

tuckeri, Monacanthus......... 78

Turbo margaritaceus......... 218

tuta, Cymatodera . . . . . . . . 344

Tylosurus acus............ 35

ardeola ............. 36

raphidoma................. 36

Typha angustifolia......... I 70

Ulæma lefroyi............. 60

Uma notata............. 3

Umbra................. 297

$\operatorname{limi} \ldots \ldots \ldots \ldots \ldots \ldots 297$

umbratilis, Notropis......... 274

Umbridæ................. 297

underwoodi, Cichlasoma....... 2 I I

undulata, Ameiva........... 193

unifasciatus, Hyporhamphus.... 36

uniformis, Cymatodera........ 344

Upeneus maculatus.......... 45

martinicus.......... 46

Uranidea.............. 337

kumlienii........... 337

urus, Ictiobus. . . . . . . . . . 249

usta, Cymatodera ...342, 343, 346, 347 ,

Uta $\ldots \ldots \ldots \ldots \ldots \ldots \ldots . \ldots \ldots$

8

graciosa............ Io

mearnsi............ 9

microscutata.......... Io

stansburiana.......... Io

vandepolli, Girardinus. . . . . . . 209

Varanus niloticus........... 409

varia, Mabuia............ 4 I I

Placopterus.......... 363

variatus, Characodon......... 155

variegatus, Coleonyx.......... 3

velifer, Carpiodes............ 25 I

venenoso apua, Mycteroperca.... 54

ventralis, Callisaurus........ 7

venustum, Enoplium.......... 384 vermiculatus, Esox........... 296

verrilli, Brosmophycis........ 86

verrucosus, Antennarius....... 88

versicolor, Nerita.......... 2 I 8

Verticaria serica........... I4

vestita, Chariessa . . . . . . . . 383

vetula, Balistes........... 76

Scarus............. jo $^{\circ}$

vetusta, Cregya.......384, 388, 390

vetustum, Enoplium......... 384

Pelonium............ 384

viduus, Clerus. . . . . . . . . 358

vigilax, Cliola............. 266

vigilis, Xantusia........... I3

Vipera hindii............ 405

virescens, Butorides.......... I94

virgata, Cardinalia .......... 2 I 8

viride, Sparisoma..... . . . . . . 72

vitreum, Stizostedion . . . . . . 3 I9

vittatus, Basiliscus. . . . . . . . . 192

vivanus, Lutianus............ 56

Vivipara..............22I

clemensæ........... $22 \mathrm{I}$

lanaoënsis ............22 I

lanaoënsis var. eta. . . . . . 22 I

lanaoënsis var. gamma.... 22I

lanaoënsis var. kappa..... 22 I

mearnsii............ 22I

pagodula . . . . . . . . . . 22 I

partelloi..............22I

vociferus, Oxyechus........... 196

volitans, Cephalacanthus....... 8I

Volvocidæ.............. 196

vulneratus, Clerus.......... 362

vulpes, Albula............ 33

wahlbergii, Ablepharus........ 4 I2

whipplii, Notropis.......... 272

wickhami, Cymatodera......354, 355

wilderi, Lampetra. . . . . . . . . 232

willardi, Crotalus............ I 8

wislizenii, Crotaphytus....... 8,9

xanthochila, Papuina........ 2 I5

xanti, Cymatodera.......... 345

Xantusia vigilis.......... I $\quad \mathbf{I}_{3}$

Xantusiida ............ 13

Xenoclerus......... $\quad 366$

edwardsii.......... . . 366

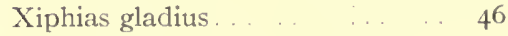








\subsection{5}

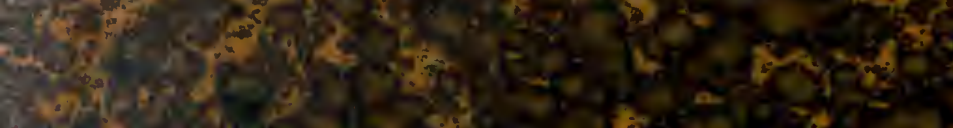

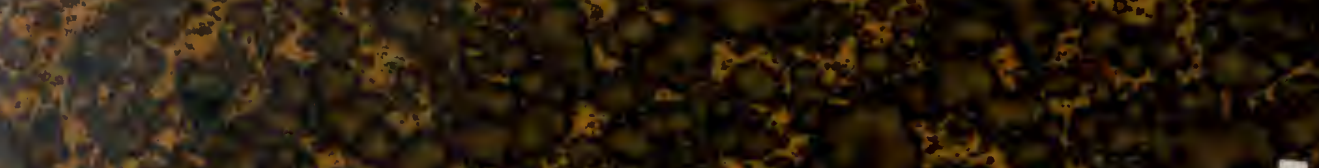

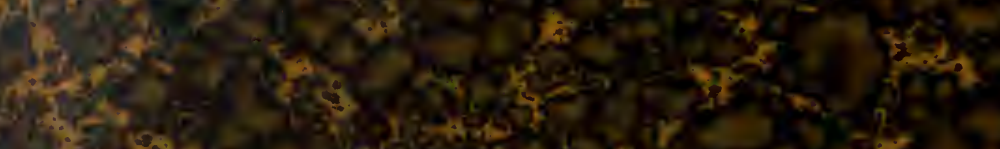
(1)

1. 0120

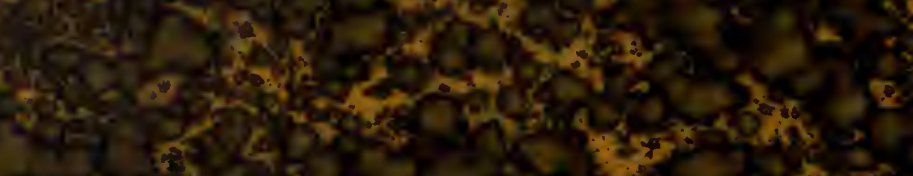

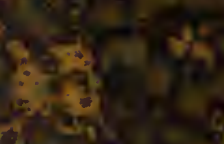

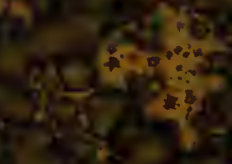

积两

4

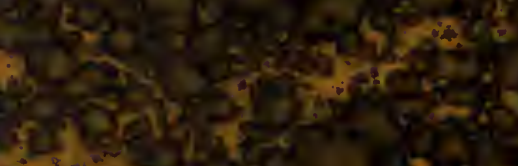

$\Phi^{3}$

m

(1) 覆 2. 3. nosio

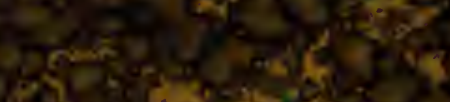
s.

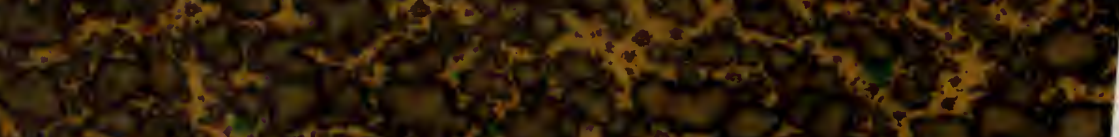

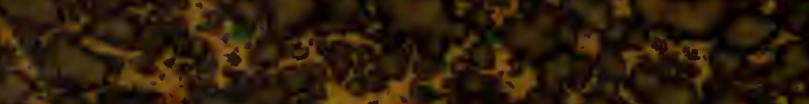

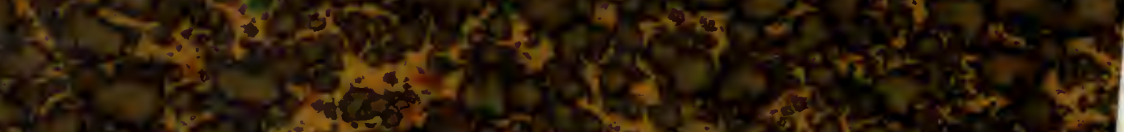

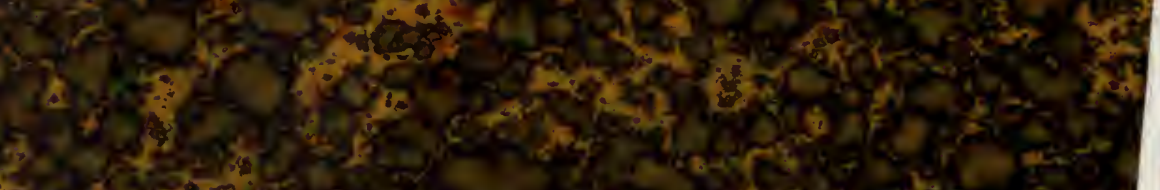
$30 x+3$ Gor

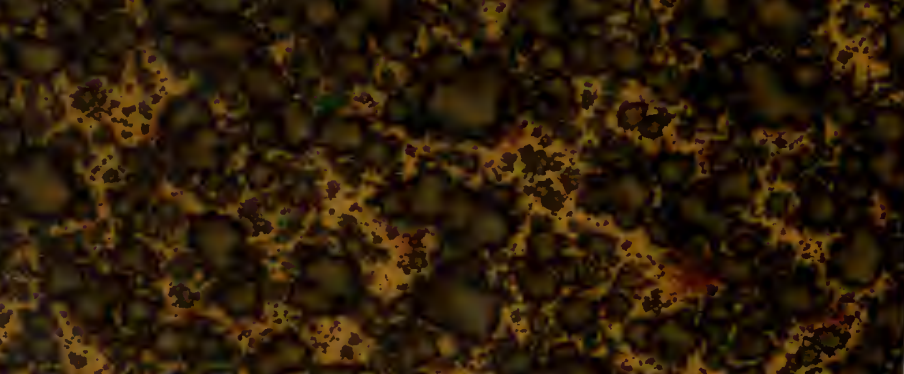
xemention

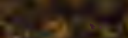

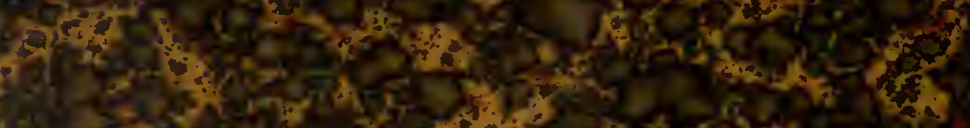

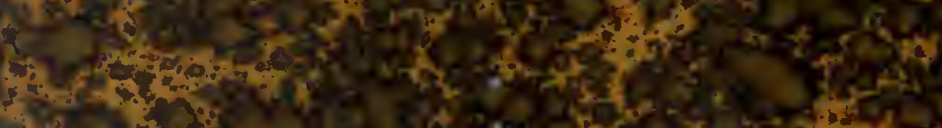

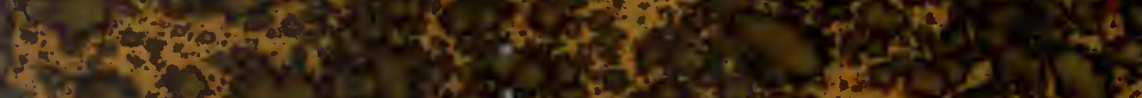

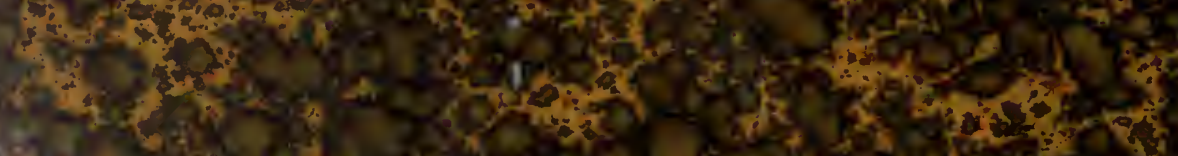

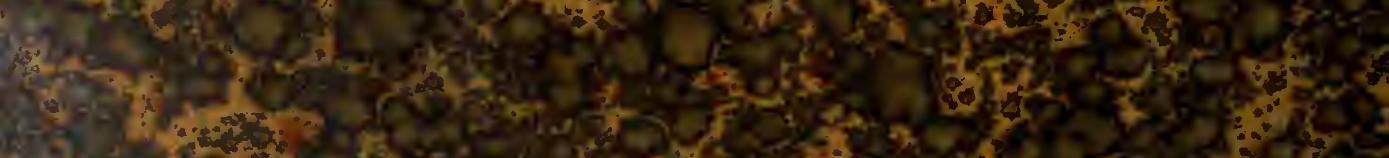





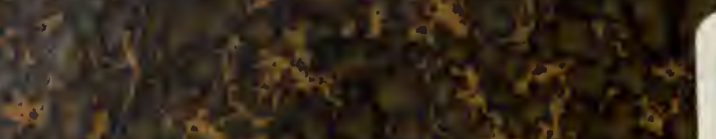

UNIVERSITY OF ILLINOIS-URBANA

590.5FI $\mathrm{COO1}$

FIELDIANA, ZOOLOGYSCHGO

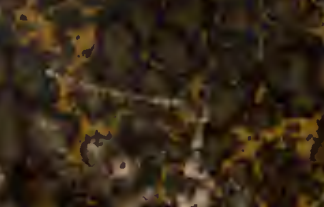

71905

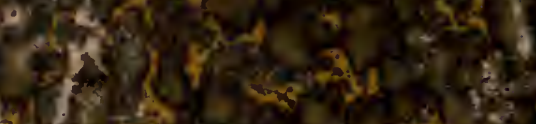

30112009379915

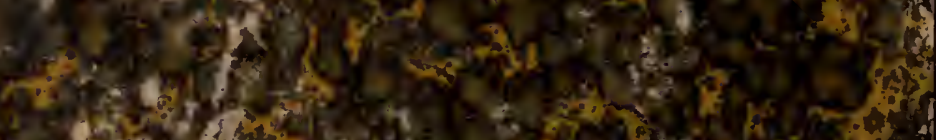

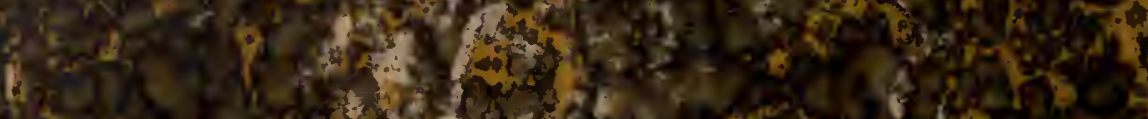

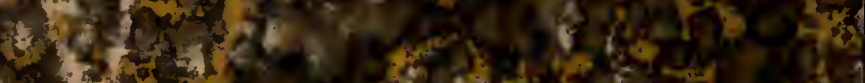

aris

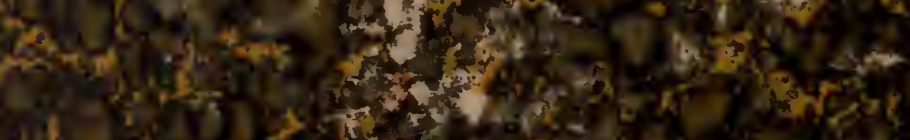

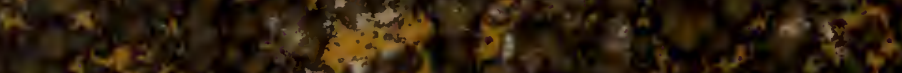

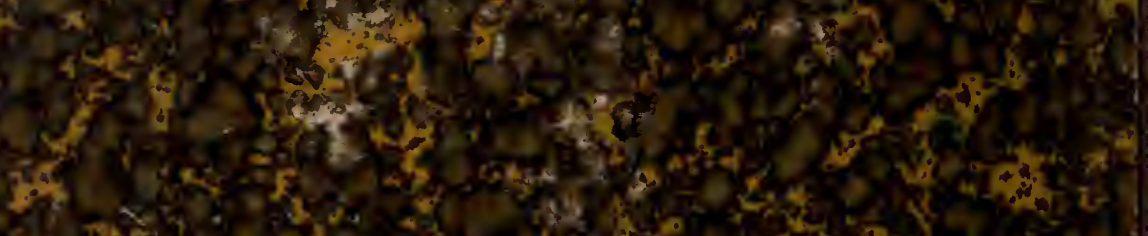

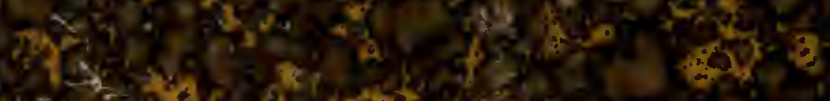

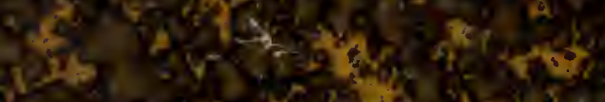

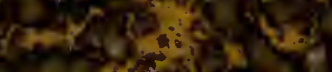

2.

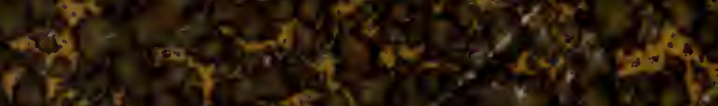

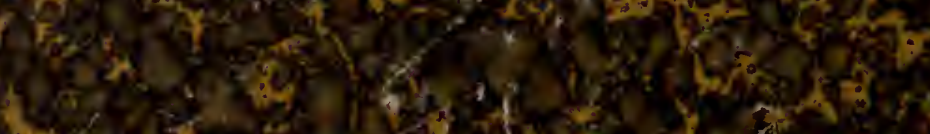

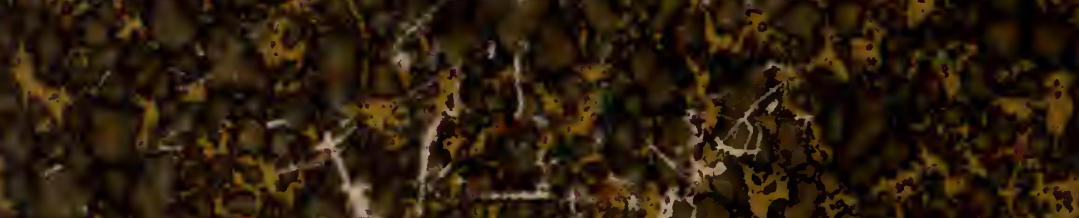

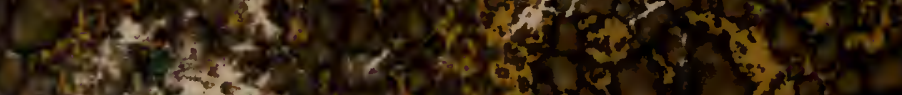

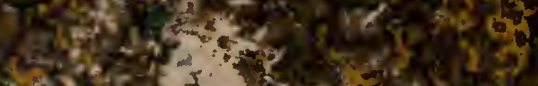
(t) 1.t. 2. 5 x 3 Non 